\title{
Supporting Information: \\ Exhaustive Conformational Sampling of Complex Fused Ring Macrocycles Using Inverse Kinematics
}

\author{
Evangelos A. Coutsias ${ }^{1, a}$, Katrina W. Lexa ${ }^{2, a}$, \\ Michael J. Wester ${ }^{3}$, Sara N. Pollock ${ }^{4}$, and Matthew P. Jacobson ${ }^{5}$ \\ ${ }^{1}$ Stony Brook University, \\ ${ }^{2}$ Merck \& Co., Inc., \\ ${ }^{3}$ University of New Mexico, \\ ${ }^{4}$ Texas A\&M University, \\ ${ }^{5}$ University of California in San Francisco, \\ ${ }^{a}$ E. A. C. and K. W. L. contributed equally to this study.
}

July 20, 2016

\section{Contents}

Section 1 discusses the procedure used to generate the starting structures for all simulations. In Section 2, we compare the performance of MMBS to BRIKARD when both are run under the parallel protocol that we consider ideal for BRIKARD. Section 3 gives a discussion of eccentricity. Section 4 is a collection of tables and figures referenced in the main text. Section 5 discusses our handling of the RMSD computation for molecules composed of identical units in symmetric arrangements, for which multiple alignments are possible. Section 6 presents a discussion of the algorithm used to sample 5 membered rings. Section 7 gives an overview of the inverse kinematics algorithms employed. In 7.1, we review basic ideas of the Triaxial Loop Closure (TLC) algorithm, while in 7.2, we present the 6-rotor/6 bar (R6B6) algorithm with a numerical example, giving the 6 alternative conformations of a 19 member ring, all but 6 of whose torsions are set to fixed values.

\section{Starting Structure Generation}

Initial coordinates of the experimental structure for each compound were downloaded from the Cambridge Structural Database (CSD), if available. Otherwise, structural data was downloaded from the PDB. This approach was followed to retain atomic naming conventions and simplify root-mean-square calculations; however, input structures could be generated from a SMILES string or by sampling in BRIKARD. Crystallographic additives and binding partners were removed, leaving only the compound of interest. Major dihedral angles along the ring were perturbed using the Coordinate Scan function of MacroModel. The resulting conformations were clustered and the two lowest-energy cluster representatives with an RMSD at least $2.5 \AA$ from the crystallographic state were selected as starting conformations. This workflow can be performed in an automated fashion, using python or Maestro's command explorer window as just two examples. We will incorporate an automated workflow accepting SMILES strings or other user input into BRIKARD for seed structure generation in the near future.

All input conformations to BRIKARD, (CF-)MMBS, and CF-LowModeMD were identical. BRIKARD's optimal implementation employs two input conformations, while the recommended implementation of the other packages requires only one. We analyzed the performance of each seed conformation and found that at the program level, neither seed structure provided an inherent advantage over the other as a starting point for sampling. Therefore, we randomly selected representative $B$ as our common seed conformation across all programs. 


\section{Parallelization Comparison}

One of BRIKARD's advantages is the ability to run it over many processors with short sampling iterations. Evaluating the performance of the current widely-applied methods under similar conditions as BRIKARD helps to highlight the fundamental difference between geometric and physical generation of the conformational ensemble. Although we cannot optimize the proprietary codes for running in parallel, we converted the published protocols into a parallel workflow run in the same manner as BRIKARD. While the physical, MD-based approach proceeds by making small variations in the structure that are bounded by an energy landscape, the geometrical method makes arbitrary changes in torsions while keeping other data invariant, such as bond angles and lengths based on the seed structure. Thus, the seed structure plays a very different role in the two approaches. For the IK method, it provides angle and bond length information to pre-minimization structures, while in the Low Mode approaches, it is the initial condition for a trajectory. Keeping these major differences in mind, we also note that for BRIKARD, generating diverse structures is easy, as it is simple to reseed a search using energy minimized structures constructed during a calculation. This may remove any biases introduced by initial angle strains.

We have carried out a comparison of BRIKARD to MMBS, both run with our protocol for BRIKARD: 40 jobs total, each for 250 iterations total, starting from two different input structures. Since CF-LowModeMD and MMBS have strong similarities in their underlying methods, comparison of BRIKARD to MMBS is sufficient. The results from running both BRIKARD and MMBS in the same fashion clearly demonstrate the improvements to sampling in this fashion are seen with BRIKARD, but not with MMBS: Figure 1. Between $69.8-97.6 \%$ of the conformations obtained via parallel, short runs of MMBS were identical across all 40 simulations. In contrast, only $0.9-27.7 \%$ of the conformations sampled by BRIKARD were identical across all 40 simulations. Furthermore, MMBS suffers from low accuracy when implemented in this way. Conformations within $0.5 \AA$ of the experimental structure were found for only $12 \%$ of the compounds examined using this approach. In contrast, running MMBS with the program's published protocol identified conformations within $0.5 \AA$ for $19.2 \%$ of the same set of compounds. Thus, although it's possible to run these programs in a manner similar to BRIKARD, this comparison indicates they should not be run in this way.

\section{Eccentricity Plot}

A comparison of the calculated eccentricity (on average) to the crystallographic eccentricity illustrates the correlation between experimental and calculated conformations: Figure 2. The plot indicates the broader spread in the eccentricity data for MMBS and CF-LowModeMD, while the averages lie closer to the fitted line for BRIKARD. The bottom right outlier corresponds to the imploded cage structure of BIX. See the main text for an in-depth discussion.

\section{Dataset Comparisons}

The set of molecules used in this study is listed in Table 1, together with the codes that we use to identify each compound in all subsequent tables and in the text. Table 2 lists molecular descriptors of size and flexibility, including cyclomatic number, number of rotatable bonds (the number of degrees of freedom sampled by BRIKARD as given by Eq. (1) in the main text), molecular weight, and eccentricity. Total wallclock time required for each run is detailed in Table 3 . The global energy minimum was identified amongst the 10,000 minimized conformations and used to determine the energy cut-off for retaining conformations within 10 or $15 \mathrm{kcal} / \mathrm{mol}$. These cut-offs were chosen because MMBS uses a $10 \mathrm{kcal} / \mathrm{mol}$ filter for reducing the total number of conformations generated, while CF-MMBS and CF-LowModeMD use a $15 \mathrm{kcal} / \mathrm{mol}$ filter. Table 4 provides the total number of conformations generated by each method for every compound and includes the number of conformations within the 10 or $15 \mathrm{kcal} / \mathrm{mol}$ filter window for BRIKARD. To contrast effective sampling around near-native states by these different techniques, full details of IP01 and IP02 torsion angle populations are given for CF-LowModeMD, MMBS, and BRIKARD overall and filtered to the low energy structures (10 or $15 \mathrm{kcal} / \mathrm{mol}$ from the minimum) in Figure 4.

One metric by which performance of BRIKARD was evaluated was the root-mean-square deviation (RMSD) of the sampled backbone conformation relative to experimental data. The RMSD to the experimental structure of all atoms along the major ring of the macrocycle was calculated using Chimera and BRIKARD's frmsd code. Only the ring atoms are used to compare to the experimental structure since the side chains, especially the polyketide tails, may adopt multiple low-energy conformations in solution. Unique conformations were judged by clustering with a heavy-atom RMSD threshold of $0.25 \AA$. For compounds composed of identical subunits (such as the cryptophane analogs), correct RMSD calculation required considering all possible alternative alignments. These ring RMSD results are provided in Table 5. 


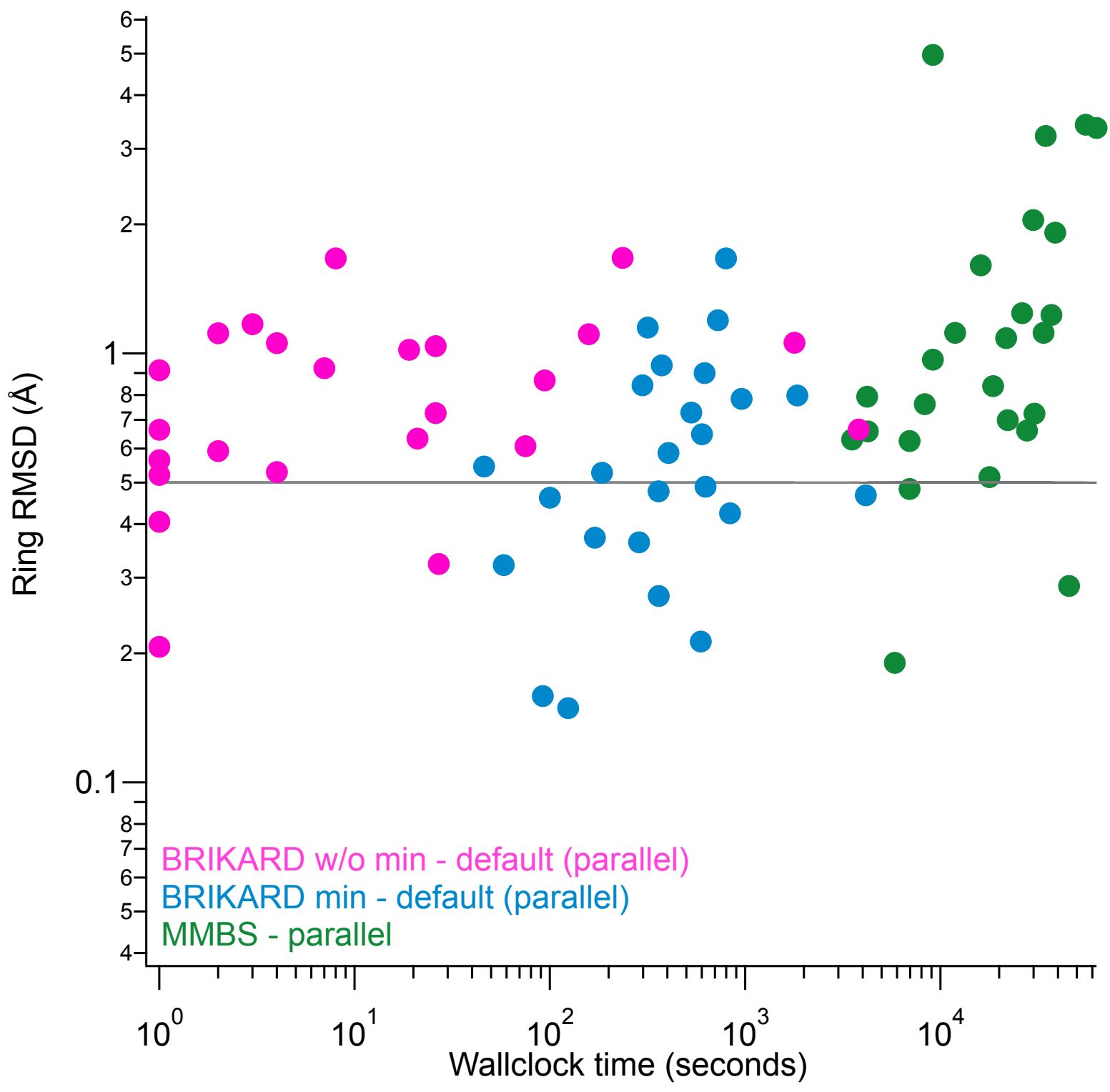

Figure 1 RMSD of ring atoms to crystallographic coordinates vs. run time according to different sampling methods. Each method was run in parallel using two different initial conformations run on 20 processors for 250 iterations each, to a total of 10,000 iterations, thus enabling comparison of parallel performance between sampling methods. 


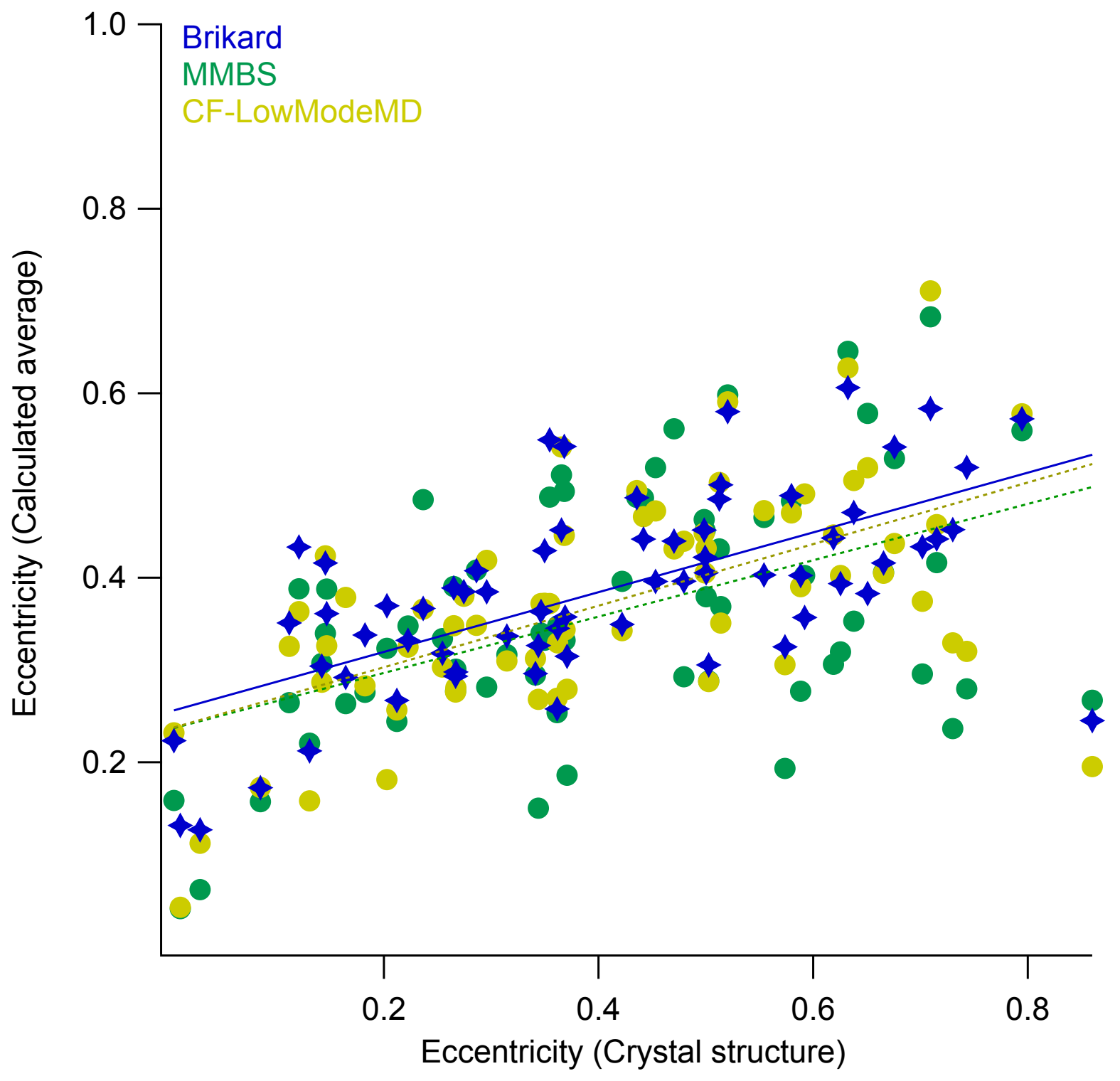

Figure 2 Calculated average eccentricity by sampling method for each macrocyclic compound versus the experimental structure eccentricity. 


\begin{tabular}{|c|c|c|}
\hline Compound & Name & Database ID (CSDS/PDB) or article source \\
\hline $1 \mathrm{D} 2$ & desosaminyl cyclododecane & $2 \mathrm{WI9}$ \\
\hline $1 \mathrm{P} 2$ & cyclic pyrrole-imidazole polyamide & $30 \mathrm{MJ}$ \\
\hline AAA & a83586c & VAFPEX \\
\hline AAS & a-Thr ascidiacyclamide & NEPMIE \\
\hline ACT & actinomycin $\mathrm{D}$ & GIDNUC \\
\hline ACX & alpha-Cyclodextrin & $2 X F Y$ \\
\hline AFB & brefeldin A & 1S9D \\
\hline AIP & AIP-III D4A & NMR from Blackwell et al. JACS v135 p18436 \\
\hline AP2 & aciculitin A & emin from Bonnet et al. JCIM 2009 v49 p2242 \\
\hline ARD & C15-R-Methylthineyl Rapamycin & 3FAP \\
\hline BC6 & 7-O-Carbamoylpremacbecin & 2VW5 \\
\hline $\mathrm{BIX}$ & cryptophane $( \pm)$-anti-1 & BIMXUR \\
\hline BKF & tacrolimus & $1 \mathrm{BKF}$ \\
\hline $\mathrm{BPH}$ & biphenyl derivative & PEWTAO \\
\hline CD4 & cryptophane $\mathrm{E}( \pm)-2[\mathrm{SbF} 6] 6$ & RAYFED \\
\hline CRP & cryptophane $\mathrm{E}$ analogue & PICKUH \\
\hline CSK & cyclosporin $\mathrm{H}$ & AMIFAE \\
\hline CY9 & cytochalasin D & $3 E K S$ \\
\hline DAP & daptomycin & $1 \mathrm{XT7}$ \\
\hline DEB & 6-deoxyerythronolide B & $1 \mathrm{Z8O}$ \\
\hline DEN & dentigerumycin & analogue, NUDWIS \\
\hline DID & didemnin B & SAFREW \\
\hline DRS & $\mathrm{N}$-terminal monocyclic product & $3 B \times S$ \\
\hline $\mathrm{E} 4 \mathrm{H}$ & 10-deoxymethynolide & 2HFK \\
\hline EPB & epothilone B & 1Q5D \\
\hline FK5 & tacrolimus & FINWEE10 \\
\hline GPGdAP & cyclo-(Glycylprolylglycyl-D-alanylprolyl) & xtal from Karle JACS v100 p1286 \\
\hline HAX & sandramycin & HAXMOI10 \\
\hline HP3 & halipeptin A & emin from Bonnet et al. JCIM 2009 v49 p2242 \\
\hline IP01 & cyclo(CLLRMRSIC) & NMR from Sillerud et al. 2003 \\
\hline IP02 & cyclo(CLLRMKSAC) & NMR from Sillerud et al. 2004 \\
\hline JZC & pochoxime B & 3INX \\
\hline KCR & hemicryptophane & KASGAO \\
\hline KET & kettapeptin & MOQLOU \\
\hline$L A B$ & latrunculin B & $2 \mathrm{~V} 52$ \\
\hline LY4 & LY333531 & $1 \mathrm{UU} 3$ \\
\hline MP4 & microcystin LR & emin from Bonnet et al. JCIM 2009 v49 p2242 \\
\hline MST & compound $5 a$ & $\begin{array}{l}\text { xtal from Mastalerz et al. Angew Chem Int Ed } 2013 \text { v52 } \\
\text { p3611 }\end{array}$ \\
\hline N1C & N1-cyclic inosine 5'-diphosphoribose & 2PGJ \\
\hline NOS & nosiheptide & NOSHEP10 \\
\hline NRB & narbomycin & $2 \mathrm{C} 7 \mathrm{X}$ \\
\hline OXE & oxytocin & $1 \mathrm{NPO}$ \\
\hline PI6 & compound 6 & $1 \mathrm{~B} 6 \mathrm{M}$ \\
\hline POL & polyoxypeptin A & NUDWIS \\
\hline PP7 & macrocycle PPi4 & $1 \mathrm{BXO}$ \\
\hline PRD & argadin & 1WAW \\
\hline PTX & pectenotoxin-2 & 2Q0R \\
\hline PXI & YC-17 & $2 \mathrm{CD} 8$ \\
\hline QN7 & bicyclic inhibitor (UK18) & 3QN7 \\
\hline RAP & rapamycin & QEKNID \\
\hline RDC & monorden & 2WER \\
\hline RDI & radicicol & $1 Q Y 8$ \\
\hline
\end{tabular}




\begin{tabular}{|c|c|c|}
\hline Compound & Name & Database ID (CSDS/PDB) or article source \\
\hline RFP & rifampicin & 2HW2 \\
\hline $\mathrm{RH} 9$ & rhizopodin & 2VYP \\
\hline S1A & soraphen & $1 \mathrm{~W} 96$ \\
\hline SFA & sanglifehrin A & 1YND \\
\hline SOR & soraphen & LAXWUC \\
\hline SP1 & sunflower trypsin inhibitor (SFTI-1) & $4 \mathrm{~K} 8 \mathrm{Y}$ \\
\hline SWI & swinholide A & $1 Y X Q$ \\
\hline TAP & tAIP-III D2A & NMR from Blackwell and et al. JACS v135 p18436 \\
\hline TET & compressed tetrahedron & $\begin{array}{l}\text { xtal from SI in Wang, Day, and Bowman-James } \\
\text { JACS } 2013 \text { v135 p392 }\end{array}$ \\
\hline TIO & thiostrepton & $1 \mathrm{E} 9 \mathrm{~W}, 3 \mathrm{CF} 5$ \\
\hline TSV & danoprevir & 3M5L \\
\hline U49 & compound 49 & 1LD8 \\
\hline WZY & spirolide C & 2WZY \\
\hline XBK & 4,5-de-epoxypimaricin & $2 \mathrm{XBK}$ \\
\hline $\mathrm{ZIO}$ & oleandomycin & 2IYA \\
\hline
\end{tabular}

Table 1: All compounds used in this study, listed alphabetically by short name, with source and full compound name. 


\begin{tabular}{|c|c|c|c|c|c|c|c|c|c|}
\hline Compound & $\begin{array}{l}\text { Bonds } \\
\text { in ring } \\
\text { system }\end{array}$ & $\begin{array}{c}\text { Cyclo- } \\
\text { matic } \\
\text { number }\end{array}$ & $\begin{array}{c}\text { Kier } \\
\text { flexibility }\end{array}$ & $\begin{array}{l}\text { Sampled } \\
\text { rings }\end{array}$ & $\begin{array}{c}\text { Rotatable } \\
\text { Bonds } \\
\text { Eq. (1) }\end{array}$ & $\begin{array}{l}\text { Atoms } \\
\text { in rings }\end{array}$ & $\begin{array}{c}\text { Molecular } \\
\text { weight }\end{array}$ & $\begin{array}{l}\text { Heavy } \\
\text { atoms }\end{array}$ & $\begin{array}{c}\text { Eccen- } \\
\text { tricity }\end{array}$ \\
\hline $1 \mathrm{D} 2$ & 18 & 2 & 9.200 & 2 & 10 & 18 & 342 & 24 & 0.630 \\
\hline 1P2 & 78 & 9 & 25.750 & 1 & 18 & 40 & 1179 & 86 & 0.710 \\
\hline AAA & 35 & 4 & 23.780 & 4 & 23 & 18 & 977 & 69 & 0.480 \\
\hline AAS & 30 & 3 & 21.130 & 1 & 18 & 10 & 793 & 54 & 0.340 \\
\hline ACT & 57 & 7 & 28.570 & 4 & 20 & 24 & 1255 & 90 & 0.240 \\
\hline ACX & 48 & 7 & 18.600 & 7 & 30 & 36 & 973 & 66 & 0.180 \\
\hline AFB & 18 & 2 & 6.430 & 2 & 7 & 16 & 280 & 20 & 0.580 \\
\hline AIP & 22 & 2 & 24.260 & 1 & 19 & 6 & 775 & 54 & 0.150 \\
\hline AP2 & 55 & 5 & 39.780 & 4 & 43 & 17 & 1353 & 96 & 0.500 \\
\hline ARD & 49 & 5 & 25.000 & 4 & 21 & 23 & 980 & 69 & 0.420 \\
\hline BC6 & 23 & 2 & 13.920 & 1 & 14 & 6 & 503 & 36 & 0.220 \\
\hline $\mathrm{BIX}$ & 111 & 13 & 26.690 & 4 & 15 & 60 & 1297 & 96 & 0.860 \\
\hline BKF & 39 & 4 & 19.950 & 4 & 21 & 18 & 804 & 57 & 0.270 \\
\hline $\mathrm{BPH}$ & 44 & 6 & 14.930 & 2 & 11 & 22 & 735 & 54 & 0.080 \\
\hline CD4 & 118 & 13 & 17.260 & 7 & 6 & 51 & 973 & 72 & 0.010 \\
\hline CRP & 85 & 10 & 18.830 & 4 & 6 & 42 & 937 & 69 & 0.030 \\
\hline CSK & 33 & 1 & 38.600 & 1 & 32 & 0 & 1203 & 85 & 0.110 \\
\hline CY9 & 26 & 4 & 9.600 & 3 & 11 & 24 & 508 & 37 & 0.550 \\
\hline DAP & 48 & 4 & 53.740 & 1 & 47 & 15 & 1620 & 72 & 0.730 \\
\hline DEB & 14 & 1 & 9.910 & 1 & 11 & 14 & 387 & 27 & 0.210 \\
\hline DEN & 43 & 5 & 19.530 & 5 & 15 & 24 & 882 & 62 & 0.700 \\
\hline DID & 38 & 4 & 30.570 & 3 & 32 & 16 & 1112 & 79 & 0.200 \\
\hline DRS & 19 & 2 & 9.530 & 2 & 7 & 6 & 362 & 26 & 0.310 \\
\hline $\mathrm{E} 4 \mathrm{H}$ & 12 & 1 & 7.790 & 1 & 8 & 12 & 296 & 21 & 0.360 \\
\hline EPB & 23 & 3 & 11.610 & 1 & 14 & 8 & 508 & 35 & 0.370 \\
\hline FK5 & 39 & 4 & 19.950 & 4 & 21 & 18 & 804 & 57 & 0.300 \\
\hline GPGdAP & 23 & 3 & 7.770 & 3 & 4 & 21 & 379 & 27 & 0.270 \\
\hline $\mathrm{HAX}$ & 12 & 3 & 32.800 & 3 & 24 & 12 & 1079 & 76 & 0.670 \\
\hline HP3 & 19 & 2 & 16.230 & 1 & 15 & 5 & 627 & 43 & 0.450 \\
\hline IP01 & 29 & 1 & 40.370 & 1 & 34 & 0 & 1088 & 73 & 0.140 \\
\hline IP02 & 29 & 1 & 37.830 & 1 & 32 & 0 & 1020 & 68 & 0.370 \\
\hline JZC & 26 & 3 & 11.980 & 2 & 10 & 24 & 443 & 32 & 0.440 \\
\hline $\mathrm{KCR}$ & 81 & 7 & 20.210 & 3 & 12 & 27 & 865 & 63 & 0.130 \\
\hline KET & 35 & 4 & 25.100 & 4 & 22 & 18 & 1007 & 71 & 0.500 \\
\hline LAB & 25 & 3 & 8.220 & 2 & 8 & 22 & 396 & 27 & 0.370 \\
\hline LY4 & 35 & 6 & 7.680 & 2 & 6 & 29 & 469 & 35 & 0.340 \\
\hline MP4 & 31 & 2 & 33.680 & 1 & 30 & 6 & 1023 & 73 & 0.350 \\
\hline MST & 226 & 29 & 38.560 & 3 & 18 & 116 & 2303 & 176 & 0.000 \\
\hline $\mathrm{N} 1 \mathrm{C}$ & 35 & 5 & 8.090 & 3 & 12 & 19 & 542 & 35 & 0.290 \\
\hline NOS & 83 & 10 & 25.520 & 2 & 18 & 40 & 1222 & 82 & 0.640 \\
\hline NRB & 20 & 2 & 12.890 & 2 & 13 & 20 & 510 & 36 & 0.590 \\
\hline OXE & 31 & 3 & 33.360 & 2 & 30 & 11 & 1064 & 73 & 0.520 \\
\hline PI6 & 27 & 3 & 16.530 & 1 & 11 & 23 & 548 & 43 & 0.620 \\
\hline POL & 42 & 5 & 20.890 & 5 & 21 & 23 & 969 & 68 & 0.720 \\
\hline PP7 & 19 & 2 & 18.060 & 1 & 16 & 18 & 639 & 44 & 0.630 \\
\hline PRD & 30 & 4 & 16.760 & 3 & 18 & 25 & 675 & 48 & 0.500 \\
\hline PTX & 56 & 9 & 12.820 & 7 & 16 & 39 & 859 & 61 & 0.510 \\
\hline PXI & 18 & 2 & 11.150 & 2 & 11 & 18 & 454 & 32 & 0.590 \\
\hline QN7 & 43 & 4 & 70.410 & 2 & 57 & 12 & 1945 & 134 & 0.500 \\
\hline RAP & 44 & 4 & 24.420 & 4 & 27 & 18 & 914 & 65 & 0.440 \\
\hline RDC & 22 & 3 & 7.740 & 2 & 7 & 19 & 344 & 25 & 0.350 \\
\hline
\end{tabular}




\begin{tabular}{|c|c|c|c|c|c|c|c|c|c|}
\hline Compound & $\begin{array}{l}\text { Bonds } \\
\text { in ring } \\
\text { system }\end{array}$ & $\begin{array}{c}\text { Cyclo- } \\
\text { matic } \\
\text { number }\end{array}$ & $\begin{array}{c}\text { Kier } \\
\text { flexibility }\end{array}$ & $\begin{array}{c}\text { Sampled } \\
\text { rings }\end{array}$ & $\begin{array}{c}\text { Rotatable } \\
\text { Bonds } \\
\text { Eq. (1) }\end{array}$ & $\begin{array}{l}\text { Atoms } \\
\text { in rings }\end{array}$ & $\begin{array}{c}\text { Molecular } \\
\text { weight }\end{array}$ & $\begin{array}{l}\text { Heavy } \\
\text { atoms }\end{array}$ & $\begin{array}{c}\text { Eccen- } \\
\text { tricity }\end{array}$ \\
\hline RDI & 22 & 3 & 8.180 & 1 & 6 & 19 & 371 & 25 & 0.350 \\
\hline RFP & 41 & 5 & 18.080 & 2 & 20 & 19 & 823 & 59 & 0.150 \\
\hline $\mathrm{RH} 9$ & 44 & 3 & 50.180 & 1 & 60 & 10 & 1470 & 104 & 0.740 \\
\hline S1A & 28 & 3 & 12.420 & 2 & 14 & 12 & 521 & 37 & 0.270 \\
\hline SFA & 44 & 5 & 29.140 & 4 & 29 & 23 & 1090 & 78 & 0.790 \\
\hline SOR & 26 & 3 & 12.420 & 2 & 13 & 12 & 521 & 37 & 0.270 \\
\hline SP1 & 43 & 4 & 44.270 & 3 & 31 & 16 & 1375 & 95 & 0.570 \\
\hline SWI & 64 & 5 & 41.250 & 5 & 54 & 24 & 1392 & 98 & 0.120 \\
\hline TAP & 22 & 2 & 17.570 & 1 & 14 & 6 & 590 & 41 & 0.250 \\
\hline TET & 124 & 9 & 38.530 & 3 & 30 & 36 & 1371 & 100 & 0.160 \\
\hline TIO & 82 & 10 & 38.090 & 3 & 33 & 41 & 1665 & 114 & 0.510 \\
\hline TSV & 36 & 6 & 15.740 & 2 & 13 & 31 & 732 & 51 & 0.470 \\
\hline U49 & 38 & 6 & 7.210 & 2 & 3 & 26 & 435 & 33 & 0.360 \\
\hline WZY & 49 & 7 & 11.160 & 7 & 11 & 31 & 694 & 50 & 0.650 \\
\hline XBK & 36 & 3 & 17.580 & 3 & 25 & 12 & 650 & 46 & 0.680 \\
\hline $\mathrm{ZIO}$ & 29 & 4 & 15.420 & 3 & 15 & 28 & 688 & 48 & 0.370 \\
\hline
\end{tabular}

Table 2: Common physiochemical descriptors for every compound in the data set. 
BRIKARD

CF-Low-

BRIKARD BRIKARD w/o mini.

Compound ModeMD MMBS CF-MMBS BRIKARD w/o mini. not ||'ed not ||'ed

\begin{tabular}{|c|c|c|c|c|c|c|c|}
\hline 1D2 & 33859 & 7740 & 9432 & 58 & 1 & 2744 & 30 \\
\hline 1P2 & 11188 & 75348 & 95760 & 317 & 2 & 14533 & 59 \\
\hline AAA & 22588 & 41040 & 51192 & 360 & 4 & 16066 & 100 \\
\hline AAS & 10658 & 23112 & 30708 & 298 & 4 & 13437 & 77 \\
\hline ACT & 22470 & 56160 & 72612 & 724 & 236 & 27750 & 5511 \\
\hline$A C X$ & 766 & 24264 & 46656 & 601 & 94 & 19722 & 1685 \\
\hline AFB & 1299 & 3168 & 3024 & 46 & 0 & 2134 & 11 \\
\hline AIP & 4009 & 32976 & 42804 & 405 & 2 & 13391 & 54 \\
\hline AP2 & 5115 & 102636 & 153396 & 836 & 21 & 45380 & 474 \\
\hline ARD & 11793 & 39312 & 65232 & 530 & 26 & 23738 & 684 \\
\hline BC6 & 29749 & 9180 & 16344 & 170 & 1 & 5352 & 35 \\
\hline $\mathrm{BIX}$ & 35176 & 59076 & 72900 & 626 & 158 & 24169 & 4216 \\
\hline BKF & 1385 & 37152 & 49284 & 473 & 8 & 15824 & 171 \\
\hline $\mathrm{BPH}$ & 17491 & 17136 & 23184 & 361 & 27 & 6037 & 851 \\
\hline CD4 & 24066 & 14004 & 13608 & 4145 & 3812 & 71914 & 47260 \\
\hline CRP & 68177 & 23472 & 23292 & 286 & 75 & 24373 & 1648 \\
\hline CSK & 377 & 87012 & 116676 & 959 & 19 & 41190 & 634 \\
\hline CY9 & 8550 & 7596 & 9648 & 124 & 1 & 3080 & 35 \\
\hline DAP & 7238 & 125244 & 231518 & 797 & 8 & 46854 & 265 \\
\hline DEB & 5208 & 5292 & 8964 & 92 & 1 & 2495 & 27 \\
\hline DEN & 9452 & 39276 & 55332 & 592 & 26 & 19116 & 511 \\
\hline DID & 23477 & 68904 & 97884 & 619 & 7 & 8006 & 206 \\
\hline DRS & 3589 & 5076 & 5940 & 1850 & 1787 & 34570 & 31780 \\
\hline $\mathrm{E} 4 \mathrm{H}$ & 734 & 2808 & 3456 & 100 & 1 & 3257 & 18 \\
\hline EPB & 21679 & 10332 & 15912 & 185 & 1 & 6133 & 39 \\
\hline FK5 & 19504 & 36612 & 47808 & 597 & 69 & 5846 & 238 \\
\hline GPGdAP & 1931 & 4464 & 3924 & 52 & 1 & 1167 & 155 \\
\hline $\mathrm{HAX}$ & 6108 & 62496 & 188676 & 371 & 3 & 17056 & 78 \\
\hline HP3 & 84106 & 22428 & 34416 & 121 & 3 & 5269 & 30 \\
\hline IP01 & 7820 & 79380 & 95832 & 1137 & 37 & 12523 & 401 \\
\hline IP02 & 36474 & 64656 & 79704 & 1027 & 30 & 11189 & 362 \\
\hline JZC & 6505 & 9792 & 15984 & 56 & 7 & 864 & 35 \\
\hline KCR & 13935 & 19764 & 19224 & 218 & 45 & 2826 & 327 \\
\hline KET & 6392 & 48420 & 66708 & 755 & 196 & 7011 & 596 \\
\hline LAB & 3204 & 4752 & 4356 & 44 & 5 & 764 & 28 \\
\hline LY4 & 2118 & 5400 & 6552 & 105 & 5 & 886 & 28 \\
\hline MP4 & 100421 & 59868 & 182052 & 370 & 8 & 10963 & 72 \\
\hline MST & 3389 & 186516 & 328680 & 2614 & 58 & 38305 & 475 \\
\hline $\mathrm{N} 1 \mathrm{C}$ & 1723 & 5436 & 5796 & 76 & 20 & 620 & 126 \\
\hline NOS & 10306 & 32184 & 56844 & 469 & 42 & 5450 & 615 \\
\hline NRB & 9733 & 11016 & 16668 & 424 & 13 & 2826 & 56 \\
\hline OXE & 20950 & 60768 & 66024 & 475 & 75 & 12152 & 591 \\
\hline PI6 & 6551 & 24660 & 35388 & 179 & 6 & 1732 & 68 \\
\hline POL & 38273 & 34668 & 64980 & 836 & 144 & 10642 & 318 \\
\hline PP7 & 1876 & 17532 & 34128 & 190 & 6 & 1548 & 36 \\
\hline PRD & 54171 & 25308 & 37224 & 557 & 11 & 3556 & 71 \\
\hline PTX & 27609 & 35208 & 37476 & 679 & 7 & 8189 & 55 \\
\hline PXI & 11382 & 10080 & 10800 & 141 & 9 & 1571 & 29 \\
\hline QN7 & 1556 & 176760 & 247716 & 11167 & 8711 & 94916 & 44258 \\
\hline RAP & 18448 & 47160 & 61740 & 954 & 80 & 16936 & 449 \\
\hline $\mathrm{RDC}$ & 298 & 3168 & 2880 & 26 & 2 & 273 & 10 \\
\hline
\end{tabular}




\begin{tabular}{|c|c|c|c|c|c|c|c|}
\hline Compound & $\begin{array}{l}\text { CF-Low- } \\
\text { ModeMD }\end{array}$ & MMBS & CF-MMBS & BRIKARD & $\begin{array}{l}\text { BRIKARD } \\
\text { w/o mini. }\end{array}$ & $\begin{array}{c}\text { BRIKARD } \\
\text { not \|'ed }\end{array}$ & $\begin{array}{l}\text { BRIKARD } \\
\text { w/o mini. } \\
\text { not ||'ed }\end{array}$ \\
\hline $\mathrm{RDI}$ & 3910 & 4356 & 4248 & 13 & 4 & 349 & 16 \\
\hline RFP & 26469 & 20268 & 32004 & 627 & 312 & 7194 & 4170 \\
\hline $\mathrm{RH} 9$ & 1676 & 128016 & 91656 & 1367 & 518 & 26903 & 3312 \\
\hline S1A & 46334 & 8712 & 14220 & 26 & 4 & 811 & 24 \\
\hline SFA & 49947 & 57348 & 101304 & 923 & 47 & 17660 & 331 \\
\hline SOR & 36358 & 11664 & 14904 & 417 & 6 & 3999 & 94 \\
\hline SP1 & 14709 & 104760 & 131256 & 2330 & 43 & 16470 & 301 \\
\hline SWI & 1197 & 133452 & 176616 & 1588 & 673 & 15250 & 4238 \\
\hline TAP & 62675 & 13212 & 20412 & 111 & 15 & 2887 & 108 \\
\hline TET & 1327 & 43092 & 53316 & 1931 & 1486 & 19461 & 6571 \\
\hline TIO & 20828 & 100692 & 141912 & 702 & 68 & 25667 & 461 \\
\hline TSV & 39088 & 26244 & 35316 & 239 & 12 & 3586 & 68 \\
\hline U49 & 526 & 3564 & 4068 & 262 & 3 & 1460 & 11 \\
\hline WZY & 38381 & 23796 & 46296 & 226 & 6 & 8312 & 42 \\
\hline XBK & 11040 & 14760 & 27360 & 477 & 6 & 3326 & 39 \\
\hline $\mathrm{ZIO}$ & 17310 & 14976 & 27432 & 293 & 9 & 5118 & 103 \\
\hline
\end{tabular}

Table 3: Wallclock run time in seconds required for full sampling by each approach used, with parallelized (||'ed) timings where allowed by algorithm. The additional CPU cost of CF-LowModeMD's multithreading procedure is between 1.4-4 times the wallclock time shown. Since BRIKARD is highly scalable, our protocol enables a significant reduction in wallclock time by distributing sampling across 40 individual processors. The maximum wallclock time elapsed when running in parallel (first two BRIKARD columns which are for parallelized runs with 250 iterations per processor) as well as the total time summed across all processors (second two BRIKARD columns which are for non-parallelized runs with 10,000 iterations total) is shown. The additional sampling time required for minimization (mini.) of structures following conformer generation by BRIKARD (first and third BRIKARD columns include minimization times) is also displayed. 


\begin{tabular}{|c|c|c|c|c|c|c|}
\hline Compound & $\begin{array}{l}\text { CF-Low- } \\
\text { ModeMD }\end{array}$ & MMBS & CF-MMBS & BRIKARD & $\begin{array}{c}\text { BRIKARD } \\
10 \mathrm{kcal} / \mathrm{mol} \\
\text { cut-off }\end{array}$ & $\begin{array}{c}\text { BRIKARD } \\
15 \mathrm{kcal} / \mathrm{mol} \\
\text { cut-off }\end{array}$ \\
\hline $1 \mathrm{D} 2$ & 4039 & 223 & 4353 & 10000 & 1497 & 3382 \\
\hline 1P2 & 8 & 235 & 1525 & 10000 & 34 & 145 \\
\hline AAA & 109 & 197 & 2801 & 10000 & 86 & 264 \\
\hline AAS & 4 & 285 & 4296 & 10000 & 88 & 306 \\
\hline ACT & 188 & 137 & 1123 & 9800 & 52 & 209 \\
\hline$A C X$ & 10 & 249 & 6465 & 10000 & 19 & 57 \\
\hline AFB & 150 & 35 & 611 & 10000 & 3400 & 6515 \\
\hline AIP & 2124 & 819 & 4354 & 10000 & 26 & 329 \\
\hline AP2 & 218 & 892 & 4323 & 10000 & 50 & 150 \\
\hline ARD & 228 & 500 & 2913 & 10000 & 166 & 500 \\
\hline BC6 & 3606 & 498 & 3419 & 10000 & 339 & 1579 \\
\hline BIX & 4892 & 138 & 2801 & 1000 & 257 & 1424 \\
\hline BKF & 23 & 696 & 4314 & 10000 & 106 & 377 \\
\hline BPH & 2016 & 58 & 1073 & 10000 & 455 & 2622 \\
\hline CD4 & 1654 & 7 & 1513 & 7163 & 65 & 256 \\
\hline CRP & 11137 & 9 & 3086 & 10000 & 28 & 73 \\
\hline CSK & 608 & 524 & 5181 & 10000 & 13 & 61 \\
\hline CY9 & 60 & 24 & 152 & 10000 & 1563 & 2811 \\
\hline DAP & 295 & 129 & 2776 & 10000 & 3 & 46 \\
\hline DEB & 1143 & 252 & 3696 & 9824 & 1016 & 2721 \\
\hline DEN & 49 & 509 & 6632 & 10000 & 34 & 87 \\
\hline DID & 1341 & 524 & 4579 & 10000 & 61 & 253 \\
\hline DRS & 904 & 197 & 2299 & 10000 & 4362 & 5062 \\
\hline $\mathrm{E} 4 \mathrm{H}$ & 116 & 18 & 271 & 10000 & 1693 & 4535 \\
\hline EPB & 3980 & 856 & 5299 & 10000 & 1312 & 3502 \\
\hline FK5 & 83 & 437 & 3500 & 10000 & 70 & 219 \\
\hline GPGdAP & 211 & 66 & 296 & 9703 & 8 & 14 \\
\hline $\operatorname{HAX}$ & 241 & 311 & 2434 & 8000 & 53 & 187 \\
\hline HP3 & 5071 & 570 & 3938 & 10000 & 244 & 695 \\
\hline IP01 & 1754 & 539 & 3124 & 10000 & 178 & 720 \\
\hline IP02 & 475 & 391 & 3309 & 10000 & 144 & 600 \\
\hline JZC & 2190 & 621 & 4996 & 10000 & 1994 & 5222 \\
\hline $\mathrm{KCR}$ & 999 & 555 & 3734 & 10000 & 3264 & 6501 \\
\hline KET & 117 & 507 & 4950 & 10000 & 99 & 376 \\
\hline LAB & 373 & 48 & 607 & 10000 & 175 & 1299 \\
\hline LY4 & 298 & 58 & 429 & 10000 & 6422 & 8763 \\
\hline MP4 & 346 & 854 & 4336 & 10000 & 35 & 180 \\
\hline MST & 447 & 117 & 4894 & 10000 & 156 & 408 \\
\hline N1C & 338 & 143 & 1943 & 10000 & 3971 & 7390 \\
\hline NOS & 495 & 317 & 3683 & 10000 & 130 & 559 \\
\hline NRB & 2367 & 94 & 1128 & 10000 & 604 & 1787 \\
\hline OXE & 145 & 573 & 4242 & 9953 & 40 & 169 \\
\hline PI6 & 6927 & 993 & 5301 & 10000 & 1786 & 4094 \\
\hline POL & 44 & 284 & 6232 & 10000 & 76 & 235 \\
\hline PP7 & 6843 & 627 & 4887 & 10000 & 174 & 915 \\
\hline PRD & 7144 & 521 & 6077 & 10000 & 478 & 2267 \\
\hline PTX & 92 & 255 & 2294 & 10000 & 246 & 954 \\
\hline PXI & 628 & 93 & 955 & 10000 & 627 & 1684 \\
\hline QN7 & 9 & 252 & 1776 & 9300 & 2 & 6 \\
\hline RAP & 232 & 676 & 4260 & 10000 & 50 & 214 \\
\hline RDC & 54 & 57 & 318 & 10000 & 5799 & 8081 \\
\hline
\end{tabular}




\begin{tabular}{lrrrrrr} 
& & & & & BRIKARD & BRIKARD \\
Compound & $\begin{array}{r}\text { CF-Low- } \\
\text { ModeMD }\end{array}$ & MMBS & CF-MMBS & BRIKARD & \multicolumn{1}{c}{$\begin{array}{c}\text { cut-off } \\
15 \mathrm{kcal} / \mathrm{mol} \\
\text { cut-off }\end{array}$} \\
\hline RDI & 1101 & 175 & 1290 & 10000 & 2350 & 5685 \\
RFP & 975 & 150 & 726 & 9800 & 562 & 1737 \\
RH9 & 260 & 137 & 1959 & 10000 & 47 & 187 \\
S1A & 1561 & 193 & 2696 & 10000 & 441 & 1802 \\
SFA & 640 & 708 & 4451 & 10000 & 53 & 284 \\
SOR & 1419 & 132 & 2103 & 10000 & 631 & 2250 \\
SP1 & 88 & 370 & 2906 & 10000 & 2141 & 385 \\
SWI & 28 & 233 & 1581 & 10000 & 0 & 336 \\
TAP & 5864 & 838 & 3180 & 10000 & 343 & 1878 \\
TET & 33 & 357 & 1496 & 9809 & 62 & 252 \\
TIO & 95 & 406 & 4727 & 9999 & 40 & 244 \\
TSV & 7953 & 507 & 3317 & 10000 & 594 & 2138 \\
U49 & 60 & 35 & 89 & 10000 & 7279 & 8501 \\
WZY & 1400 & 177 & 1858 & 9978 & 65 & 334 \\
XBK & 2102 & 564 & 5523 & 10000 & 45 & 259 \\
ZIO & 338 & 222 & 2732 & 9800 & 143 & 465
\end{tabular}

Table 4: Number of conformations generated by each method. The number of conformations within a $10 \mathrm{kcal} / \mathrm{mol}$ or $15 \mathrm{kcal} / \mathrm{mol}$ window of the lowest-energy conformation sampled by BRIKARD are also provided to faciliate comparison with MMBS, CF-MMBS, and CF-LowModeMD. 
CF-Low-

BRIKARD

Compound ModeMD MMBS CF-MMBS BRIKARD w/o minimization

\begin{tabular}{|c|c|c|c|c|c|}
\hline 1D2 & 0.33 & 0.63 & 0.39 & 0.34 & 0.41 \\
\hline 1P2 & 2.20 & 0.86 & 0.80 & 0.83 & 1.15 \\
\hline AAA & 0.70 & 1.00 & 0.91 & 0.50 & 0.53 \\
\hline AAS & 2.20 & 0.49 & 0.42 & 0.84 & 1.06 \\
\hline ACT & 2.09 & 2.96 & 0.94 & 1.20 & 1.67 \\
\hline$A C X$ & 0.73 & 0.34 & 0.30 & 0.62 & 0.87 \\
\hline AFB & 0.62 & 0.74 & 0.69 & 0.55 & 0.54 \\
\hline AIP & 0.58 & 1.06 & 0.81 & 0.58 & 0.59 \\
\hline AP2 & 3.29 & 0.57 & 0.59 & 0.39 & 0.63 \\
\hline ARD & 0.94 & 0.24 & 0.20 & 0.37 & 1.04 \\
\hline BC6 & 0.14 & 0.55 & 0.47 & 0.37 & 0.91 \\
\hline BIX & 0.84 & 1.12 & 0.33 & 0.49 & 1.11 \\
\hline BKF & 0.87 & 0.63 & 0.62 & 0.61 & 0.56 \\
\hline $\mathrm{BPH}$ & 4.12 & 0.59 & 0.50 & 0.30 & 0.32 \\
\hline CD4 & 4.44 & 4.55 & 4.40 & 0.33 & 0.67 \\
\hline CRP & 2.61 & 3.10 & 2.87 & 0.27 & 0.61 \\
\hline CSK & 2.52 & 0.61 & 0.48 & 1.00 & 1.02 \\
\hline CY9 & 0.46 & 0.47 & 0.48 & 0.15 & 0.21 \\
\hline DAP & 2.18 & 4.25 & 4.34 & 1.48 & 1.67 \\
\hline DEB & 0.11 & 0.13 & 0.13 & 0.13 & 0.52 \\
\hline DEN & 0.38 & 0.94 & 0.85 & 0.33 & 0.73 \\
\hline DID & 0.43 & 1.14 & 1.13 & 0.80 & 0.92 \\
\hline DRS & 0.75 & 0.64 & 0.51 & 0.80 & 1.06 \\
\hline $\mathrm{E} 4 \mathrm{H}$ & 0.64 & 0.72 & 0.65 & 0.46 & 0.56 \\
\hline EPB & 0.62 & 0.66 & 0.59 & 0.58 & 0.66 \\
\hline FK5 & 0.48 & 0.28 & 0.69 & 0.77 & 1.18 \\
\hline GPGdAP & 0.13 & 0.10 & 0.10 & 0.50 & 0.67 \\
\hline $\mathrm{HAX}$ & 1.19 & 2.53 & 2.27 & 0.94 & 1.17 \\
\hline HP3 & 0.14 & 0.11 & 0.54 & 0.06 & 0.39 \\
\hline IP01 & 0.73 & 3.03 & 4.23 & 0.83 & 0.83 \\
\hline IP02 & 1.21 & 4.15 & 4.04 & 0.93 & 0.82 \\
\hline JZC & 0.50 & 0.26 & 0.25 & 0.42 & 0.63 \\
\hline KCR & 2.01 & 2.10 & 2.08 & 0.57 & 0.63 \\
\hline KET & 0.71 & 1.37 & 0.79 & 0.17 & 0.48 \\
\hline LAB & 0.66 & 0.44 & 0.43 & 0.12 & 0.71 \\
\hline LY4 & 0.12 & 0.12 & 0.12 & 0.13 & 0.24 \\
\hline MP4 & 0.46 & 0.55 & 0.49 & 0.73 & 0.76 \\
\hline MST & 1.53 & 1.30 & 1.20 & 0.42 & 1.31 \\
\hline $\mathrm{N} 1 \mathrm{C}$ & 0.39 & 0.47 & 0.47 & 0.48 & 0.47 \\
\hline NOS & 0.41 & 0.93 & 0.57 & 1.01 & 1.18 \\
\hline NRB & 0.24 & 0.37 & 0.24 & 0.22 & 0.40 \\
\hline OXE & 0.68 & 0.70 & 0.62 & 0.48 & 0.65 \\
\hline PI6 & 0.75 & 0.43 & 0.21 & 0.16 & 0.43 \\
\hline POL & 0.85 & 1.38 & 1.20 & 0.23 & 0.51 \\
\hline PP7 & 0.37 & 0.52 & 0.28 & 0.23 & 0.30 \\
\hline PRD & 0.15 & 0.47 & 0.06 & 0.04 & 0.26 \\
\hline PTX & 0.54 & 0.28 & 0.25 & 0.53 & 0.69 \\
\hline PXI & 0.28 & 0.22 & 0.22 & 0.21 & 0.22 \\
\hline QN7 & 3.83 & 3.36 & 6.12 & 1.66 & 1.72 \\
\hline RAP & 0.88 & 0.35 & 0.30 & 0.72 & 1.11 \\
\hline RDC & 0.47 & 0.46 & 0.38 & 0.10 & 0.29 \\
\hline RDI & 0.41 & 0.39 & 0.67 & 0.39 & 0.58 \\
\hline
\end{tabular}




\begin{tabular}{lccccc} 
Compound & $\begin{array}{c}\text { CF-Low- } \\
\text { ModeMD }\end{array}$ & MMBS & CF-MMBS & BRIKARD & $\begin{array}{c}\text { BRIKARD } \\
\text { w/o minimization }\end{array}$ \\
\hline RFP & 0.71 & 1.71 & 1.26 & 1.06 & 1.04 \\
RH9 & 1.78 & 2.80 & 1.52 & 1.24 & 1.36 \\
S1A & 0.39 & 0.49 & 0.53 & 0.47 & 0.50 \\
SFA & 0.32 & 0.55 & 0.37 & 0.47 & 0.69 \\
SOR & 0.46 & 0.49 & 0.47 & 0.43 & 0.47 \\
SP1 & 2.34 & 2.39 & 2.16 & 1.51 & 1.46 \\
SWI & 1.46 & 1.74 & 1.65 & 1.37 & 1.83 \\
TAP & 0.25 & 0.63 & 0.36 & 0.30 & 0.37 \\
TET & 2.31 & 2.12 & 2.12 & 1.87 & 1.88 \\
TIO & 2.13 & 3.33 & 2.69 & 0.26 & 0.63 \\
TSV & 0.64 & 0.72 & 0.67 & 0.10 & 0.42 \\
U49 & 0.47 & 0.43 & 0.43 & 0.45 & 0.45 \\
WZY & 0.17 & 0.35 & 0.28 & 0.14 & 0.72 \\
XBK & 0.42 & 0.43 & 0.41 & 0.36 & 0.63 \\
ZIO & 0.35 & 0.35 & 0.39 & 0.31 & 0.56
\end{tabular}

Table 5: Lowest ring RMSD to experiment. 


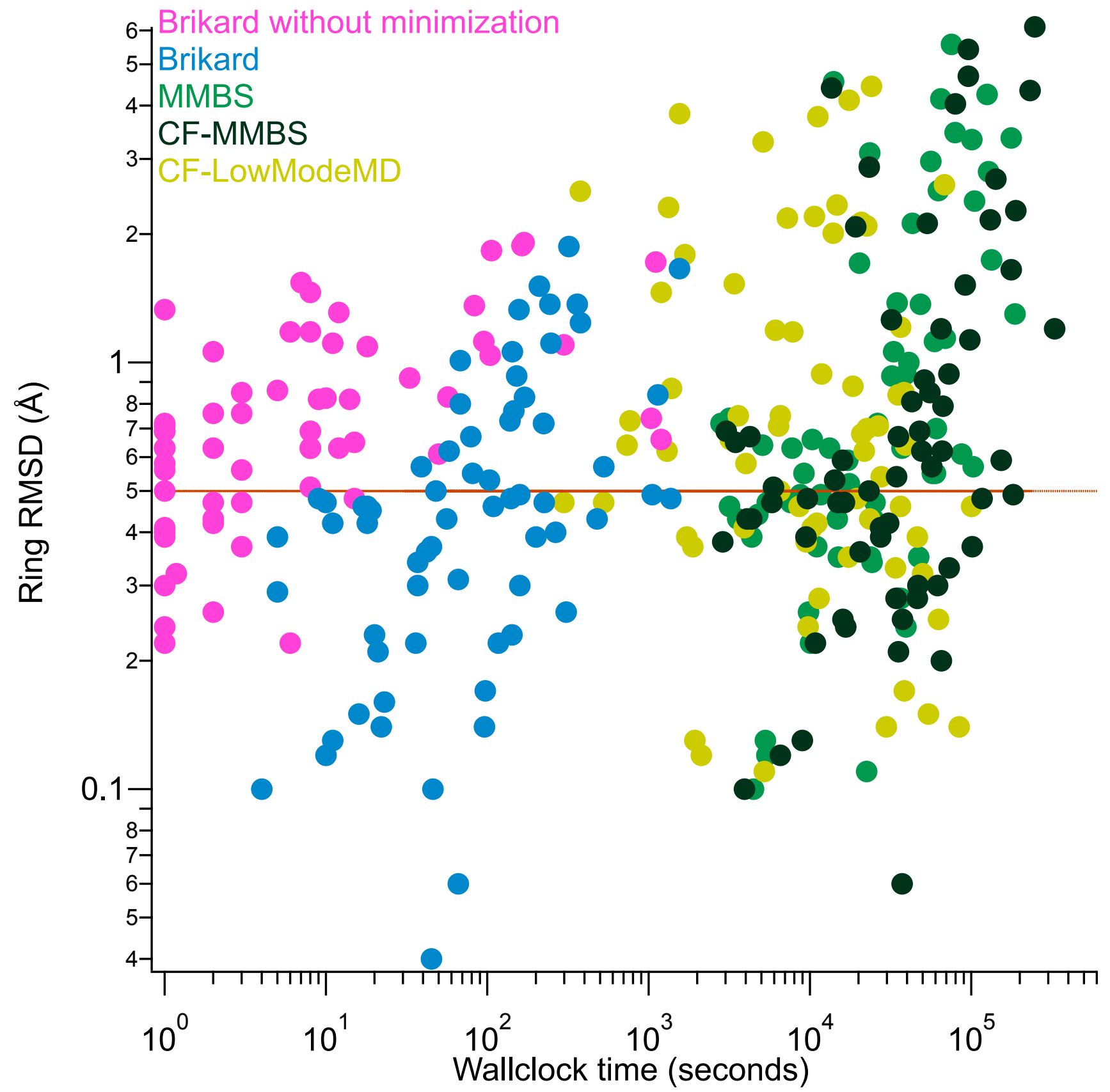

Figure 3 RMSD of ring atoms to crystallographic coordinates vs. run time for the full data set, according to different sampling methods. 

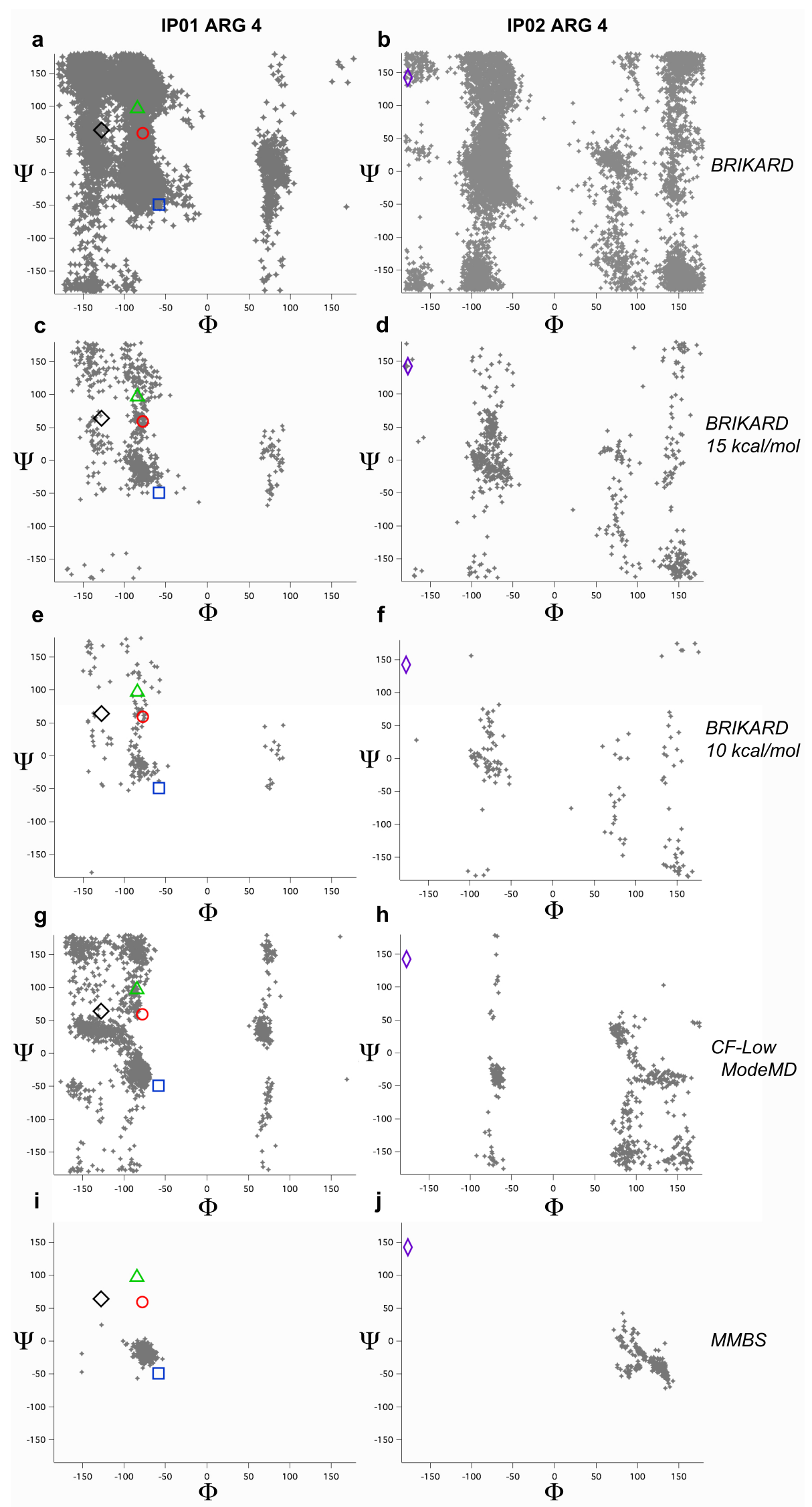

IP01 states: A $(\diamond), B(\square)$, C $(\Delta)$, D (०) IP02 state $(\diamond)$

Figure 4 Arginine-4 torsional angle sampling across the potential energy surfaces of IP01 and IP02 by a-b) BRIKARD, $c-d)$ conformations from BRIKARD restricted to within $15 \mathrm{kcal} / \mathrm{mol}$ of the lowest energy identified, e-f) conformations from BRIKARD restricted to within $10 \mathrm{kcal} / \mathrm{mol}$ of the lowest energy identified, g-h) CF-LowModeMD (protocol applies a $15 \mathrm{kcal} / \mathrm{mol}$ cut-off), and i-j) MMBS (default protocol applies a $10 \mathrm{kcal} / \mathrm{mol}$ cut-off). 


\section{RMSD Calculations: Symmetry}

The calculation of the Root Mean Square Distance (RMSD) between two conformations of a molecule involves two main steps, alignment and optimal superposition. Aligning two conformations means establishing a 1-1 correspondence between equivalent atoms in each conformation-that is, a correspondence between identical molecular graphs. Optimal superposition is found by rotating and translating one structure so that the sum of the squares of the distances between equivalent points in the two structures is minimized. The optimal translation simply superimposes the barycenters of the two point sets, while the optimal rotation requires solving a $4 \times 4$ eigenvalue problem for the quaternion giving the optimal rotation about the common barycenter ${ }^{1}$.

When the molecule is composed of identical subunits in an arrangement with a symmetric graph, as was the case for 1P2, AAS, ACX, BIX, BPH, CD4, CRP, HAX, KCR, MST, RH9, SWI and TET (shown in Figure 5 colored to highlight identical building blocks for each), then alternative equivalent alignments may be possible, leading to possibly lower RMSD. The requirement for this to occur is that the reindexed molecular graph is equivalent to the original. Here, ACX, MST and TET are hexamers, while BIX, CD4, CRP and KCR are trimers, and 1P2, AAS, BPH, HAX, RH9 and SWI are dimers. Of these, MST, TET, BIX, CD4 and CRP are composed of subunits with palindromic symmetry.

More precisely, the graphs of MST and TET have tetrahedral symmetry, so that 24 distinct alignments are possible, while ACX has cyclic symmetry of order 6 . All the dimers have cyclic symmetry of order two. Among the trimers, KCR has cyclic symmetry of order 3 , while the others (BIX, CD4, CRP) have full dihedral symmetry of order 3 , and may be aligned "upside-down", giving a total of 6 possible alignments. The results shown in SupportingTable 5 for each of the above compounds and all methods were based on carrying out all possible alignments. Invariably, we found that a lower RMSD was possible for some alignment other than the original, and in a few cases a much lower RMSD was found. This is of course expected, as including all alternative alignments effectively multiplies the ensemble size by a factor equal to the order of symmetry of a given compound. 

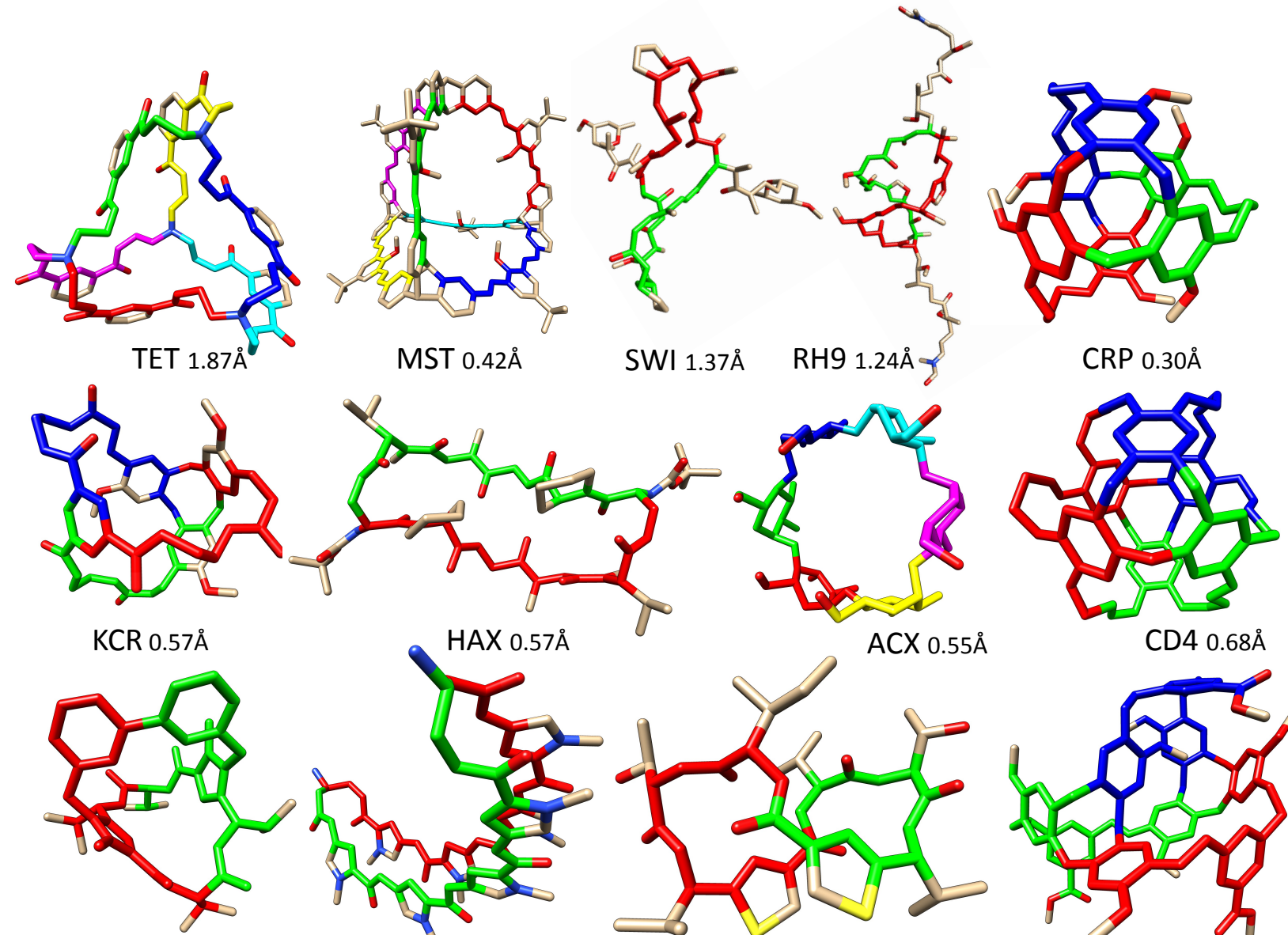

BPH $0.48 \AA$

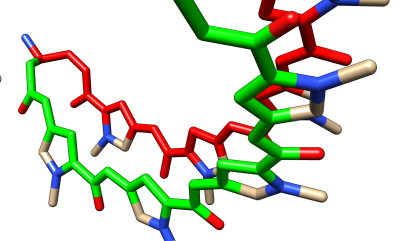

$1 \mathrm{P} 21.33 \mathrm{~A}$

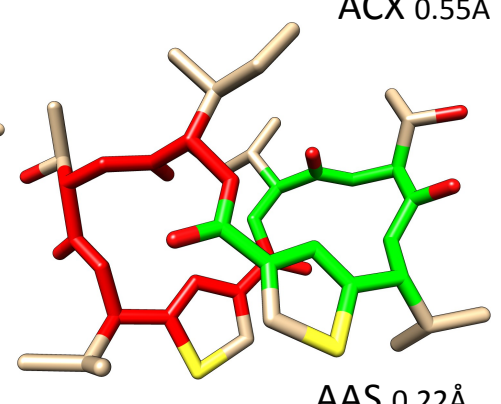

AAS $0.22 \AA$

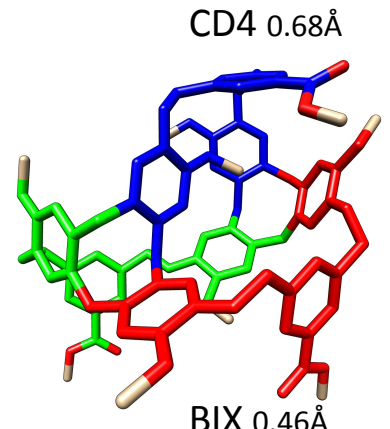

$\mathrm{BIX} 0.46 \AA$

Figure 5 The symmetric molecules in the dataset. The colored components depict the main backbone atoms included in the RMSD computation from each identical building block. Best RMSD fits found by BRIKARD are shown. 


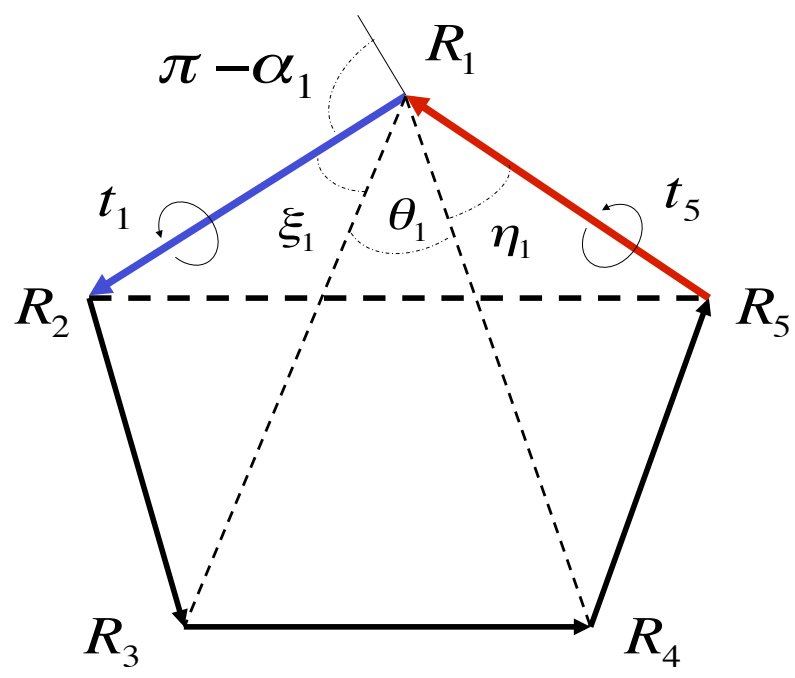

Figure 6 Modeling the 5-member ring as a spherical Cardan linkage.

\section{Five Membered Rings}

We employ a purely algebraic (polynomial) version of the algorithm discussed in ${ }^{12}$. A seed set of 5-membered ring structures is generated by the Bricard tetrahedral equation ${ }^{5}$. This construction relies on deforming two of the ring angles to achieve mobility as a function of one of the torsions. This approach was shown $\mathrm{in}^{12}$ to constitute a reasonable approximation for the conformational states of the pyrrolidine ring. We employ this approach here to derive a sample for the two pucker states of a nonplanar 5-member ring as a function of one of its torsions.

Referring to Figure 6, consider the 5-membered ring as a tetrahedral angle $O A_{1} A_{2} A_{3} A_{4}$ with apex at $O=\mathbf{R}_{1}$ and vertices $A_{1}=\mathbf{R}_{2}, A_{2}=\mathbf{R}_{3}, A_{3}=\mathbf{R}_{4}, A_{4}=\mathbf{R}_{5}$. We are justified to do so since the angles from the vertex to any two atoms that are consecutive along the ring are fixed, as is the angle $\angle\left(A_{1}, O, A_{4}\right)$. More succinctly, we consider the motions of the 5 -member ring that preserve all edges lengths as well as the 3 bond angles at $\mathbf{R}_{5}, \mathbf{R}_{1}, \mathbf{R}_{2}$. We consider the relation between the dihedral angles $t_{1}, t_{5}$ with half tangents $u=\tan \frac{t_{1}}{2}, v=\tan \frac{t_{5}}{2}$, resulting in the tetrahedral equation

$$
A u^{2} v^{2}+B u^{2}+2 C u v+D v^{2}+E=0
$$

where

$$
\begin{aligned}
& A=\cos \theta_{1}-\cos \left(\alpha_{1}+\xi_{1}+\eta_{1}\right), B=\cos \theta_{1}-\cos \left(\alpha_{1}+\xi_{1}-\eta_{1}\right) \\
& D=\cos \theta_{1}-\cos \left(\alpha_{1}-\xi_{1}+\eta_{1}\right), E=\cos \theta_{1}-\cos \left(\alpha_{1}-\xi_{1}-\eta_{1}\right) \\
& C=-2 \sin \xi_{1} \sin \eta_{1} .
\end{aligned}
$$

Finally, we can solve Eq. (1) for $v$ in terms of $u$

$$
v=\frac{-C u \pm \sqrt{C^{2} u^{2}-\left(A u^{2}+D\right)\left(B u^{2}+E\right)}}{A u^{2}+D}
$$

To each value of $t_{1}$ there correspond two possible values of $t_{2}$ and also of $\alpha_{3}, \alpha_{4}$. The latter are determined uniquely once a sign for $v$ has been chosen. For typical 5-membered rings, the variation of these angles inherent in this model is within the physically acceptable range of $5^{\circ}-10^{\circ}$. Random conformations for $t_{1}=2 \tan ^{-1} u$ are found by randomly sampling $u$ from the interval $u_{-} \leq u \leq u_{+}$where $u_{ \pm}$are the roots of the discriminant:

$$
C^{2} u^{2}-\left(A u^{2}+D\right)\left(B u^{2}+E\right) \geq 0 .
$$




\section{Inverse Kinematic Algorithms}

\subsection{The Triaxial Method}

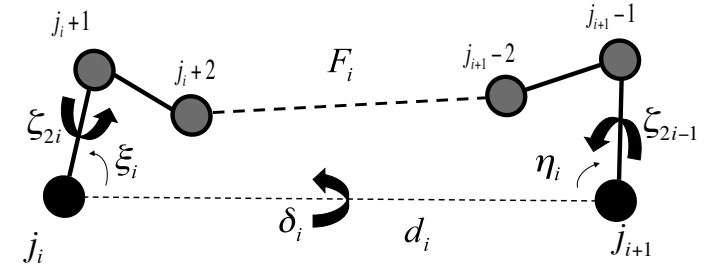

(a)

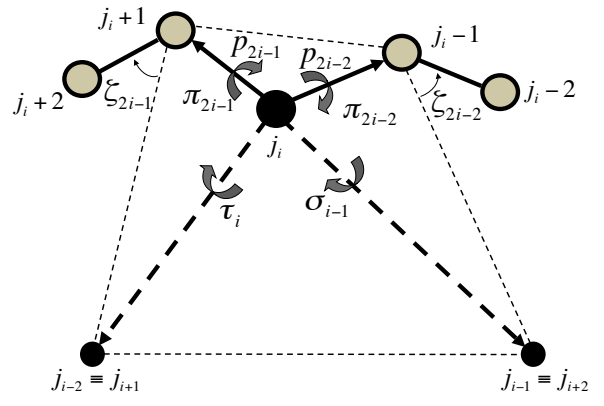

(b)

Figure 7 The basic units of TLC: (a) a segment, showing only essential atoms, (b) the joining of two segments at a vertex.

We have described different implementations of the TLC method previously ${ }^{2-4}$. The implementation presented here involves full algebraic expressions for the chain torsions in terms of the triaxial variables which have not appeared previously. We will refer to our previous work for basic equation derivations and focus on presenting the complete set of equations used to determine the pivot torsions in terms of the remaining variables, i.e., bond lengths and bond angles, $b_{i}, \theta_{i}, i=1, \ldots, N$, and the driver torsions $q_{i}, i=1, \ldots, N-6$. Let the ring atoms be numbered consecutively from 1 to $N$, with $N$ being connected to 1 to form a closed cycle. We assume that 3 atoms may be found along the chain, denoted as $A, B, C$, such that the two bonds connecting each to its neighbors are rotatable (and hence available for sampling). To ensure non-overlap, we demand that the indices (with the correspondence $i_{4}=i_{1}$ ) satisfy $2 \leq i_{1}+1<i_{2}<i_{3}-1<N+i_{1}-2$, that is, each of $A, B, C$ are separated by one or more atoms. Given values of the $b_{i}, \theta_{i}, q_{i}$, we then seek values of the $p_{j}, j=1, \ldots, 6$ that result in a closed chain of correct geometry (i.e., all other DoF are as specified). The pairing of the pivots allows a key simplification: the chain may be partitioned into 3 segments, $A B, B C$ and $C A$, whose geometry is fully determined by the given values of the $q$-torsions (and bond lengths and angles) between their endpoints. As the length of each segment is determined, the triangle $A B C$ (Figure 8 ) is known, and the only remaining indeterminacy is the orientation of each fragment about the corresponding edge of the triangle $A B C$. We thus seek the angle $\tau_{i}$ (where $\tau_{1}=\angle(C A B b), \tau_{2}=\angle(A B C c), \tau_{3}=\angle(B C A a)$ ) of rotation of each fragment about its corresponding edge that orients the end bonds at each junction so that the angles between them assume the prescribed values. The problem has thus been reduced to finding three unknown angles subject to three constraints. We introduce dihedrals (Figure $7(\mathrm{~b})) \sigma_{i}=\angle\left(j_{i-1}, j_{i}, j_{i+1}, j_{i+1}-1\right.$ ) and note that (Figure 7(a)) $\sigma_{i}=\tau_{i}+\delta_{i}$ with $\delta_{i}=\angle\left(j_{i}+1, j_{i}, j_{i+1}, j_{i+1}-1\right)$. We can determine the pivot torsions by solving a system of three biquadratic polynomials in the variables $u_{i}=\tan \tau_{i} / 2$. The system can be written in the form of three biquadratic tetrahedral equations ${ }^{5}$ :

$$
F\left(v_{i}, u_{i+1} ; \alpha_{i}, \theta_{i}, \xi_{i}, \eta_{i}\right)=0, v_{i}=\frac{u_{i}+\Delta_{i}}{1-\Delta_{i} u_{i}}, i=1,2,3\left(u_{4}=u_{1}\right) .
$$

Here, the apical angles $\alpha_{i}, \theta_{i}, \xi_{i}, \eta_{i}$ at the pivot vertex $i$ are defined as the angles $\xi_{i}=\angle\left(j_{i}+1, j_{i}, j_{i+1}\right), \eta_{i}=\angle\left(j_{i}, j_{i+1}, j_{i+1}-\right.$ 1) (Figure $7(\mathrm{a})), \theta_{i}=\angle\left(j_{i}-1, j_{i}, j_{i}+1\right), \alpha_{i}=\angle\left(j_{i-1}, j_{i}, j_{i+1}\right.$ (Figure $\left.7(\mathrm{~b})\right)$. The dihedral between the first and last bond in a fragment, $\delta_{i}=\angle\left(j_{i+1}-1, j_{i+1}, j_{i}, j_{i}+1\right)$ connects $\sigma_{i}$ to $\tau_{i}$ :

$$
\sigma_{i}=\tau_{i}+\delta_{i} \Rightarrow v_{i}=\frac{u_{i}+\Delta_{i}}{1-\Delta_{i} u_{i}}, \quad \Delta_{i}=\tan \frac{\delta_{i}}{2} .
$$

Eliminating the $v_{i}$, we arrive at a system of three polynomials in the $u_{i}$,

$$
G\left(u_{i}, u_{i+1} ; \alpha_{i}, \theta_{i}, \xi_{i}, \eta_{i}, \delta_{i}\right)=0, i=1,2,3\left(u_{4}=u_{1}\right)
$$




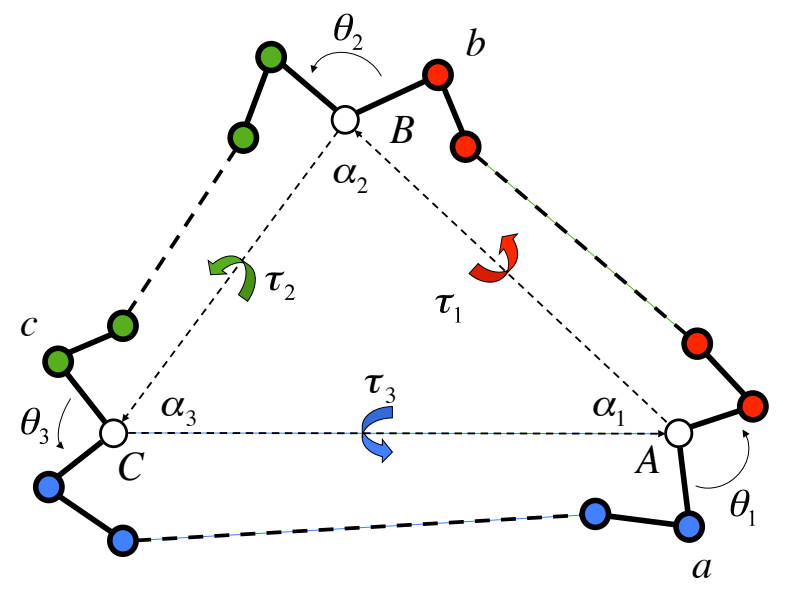

Figure 8 Schematic of TLC, showing key atoms about each pivot.

Once the system for the $u_{i}$ has been solved, each of the (up to 16) solutions is converted to the complementary torsions at the vertices $\pi_{i}$, and these are then converted to backbone torsions (Figure 7(b))

$$
p_{i}=\pi_{i}+\zeta_{i}, i=1, \ldots, 6 .
$$

\subsubsection{The Tetrahedral: Complete Solution}

A rather straightforward, but important issue is to create a fully algebraic formulation for computing the torsions about the actual rotor axes in the TLC method. As the method involves the $\tau_{k}, k=1,3$ torsions about the edges of the virtual triangle formed by the three points of confluence of the rotors, these need to be converted to the six torsions $p_{j}, j=1, \ldots, 6$. Earlier implementations of the TLC algorithm ${ }^{2-4}$ perform this conversion through constructing the closed ring or loop from the $\tau_{k}$ and then measuring the actual values of the $p_{j}$ from the Cartesian coordinates, thus using transcendental evaluations. We give here a geometrical analysis of the tetrahedral angle that shows how this may be done in a purely algebraic fashion. This is important for further exploiting the benefits of the algebraic formulation, and contributes a small speedup to the algorithm as well. No analogous step is needed for the R6B6 algorithm, since it operates directly on the torsions about the rotors, albeit in the Denavit-Hartenberg frame, as discussed in the following section.

Referring to Figure 9, consider a tetrahedral angle $O A B C D$ with apex at $O$ and vertices $A, B, C, D$. The tetrahedral is fully specified (choosing between two sides) by selecting an orientation of the vertices. In this way, we identify positive orientation of the facets with the choice of the dihedral angles $\phi, \psi, \omega, \chi: \phi$ is the angle of rotation of plane $O D A$ about oriented edge $O A$ into plane $O A B ; \psi$ is the angle of rotation of plane $O A B$ about oriented edge $O B$ into plane $O B C ; \omega$ is the angle of rotation of plane $O B C$ about oriented edge $O C$ into plane $O C D$; and $\chi$ is the angle of rotation of plane $O C D$ about oriented edge $O D$ into plane $O D A . \alpha, \beta, \gamma, \delta$ are the angles of the facets $A O B, B O C, C O D, D O A$ at the apex $O$ (apical angles). We will give below the formulas for the dihedrals $\psi, \omega, \chi$ in terms of the dihedral $\phi$ and the four apical angles. We now introduce the half tangents of the dihedrals: $u=\tan \frac{\phi}{2}, v=\tan \frac{\psi}{2}, w=\tan \frac{\omega}{2}, x=\tan \frac{\chi}{2}$, with $\cos \phi=\frac{1-u^{2}}{1+u^{2}}, \sin \phi=\frac{2 u}{1+u^{2}}$, etc., then the following relationship holds between the pair of adjacent dihedrals $(\phi, \psi)$ :

$$
(\phi, \psi): \quad A u^{2} v^{2}+B u^{2}+2 C u v+D v^{2}+E=0
$$

where

$$
\begin{aligned}
A=\cos \gamma-\cos (\alpha+\beta+\delta), & B=\cos \gamma-\cos (\alpha+\beta-\delta) \\
D=\cos \gamma-\cos (\alpha-\beta+\delta), & E=\cos \gamma-\cos (\alpha-\beta-\delta) \\
C= & -2 \sin \beta \sin \delta .
\end{aligned}
$$


In addition, we have the relationships between pairs of opposite dihedrals:

$\cos (B O D)=\cos \alpha \cos \delta+\cos \phi \sin \alpha \sin \delta=\cos \beta \cos \gamma+\cos \omega \sin \beta \sin \gamma$

$\cos (A O C)=\cos \alpha \cos \beta+\cos \psi \sin \alpha \sin \beta=\cos \gamma \cos \delta+\cos \chi \sin \gamma \sin \delta$

In terms of half tangents, these become

$$
\begin{aligned}
& L_{1}+\frac{1-u^{2}}{1+u^{2}} M_{1}=L_{3}+\frac{1-w^{2}}{1+w^{2}} M_{3} \\
& L_{2}+\frac{1-v^{2}}{1+v^{2}} M_{2}=L_{4}+\frac{1-x^{2}}{1+x^{2}} M_{4}
\end{aligned}
$$

with

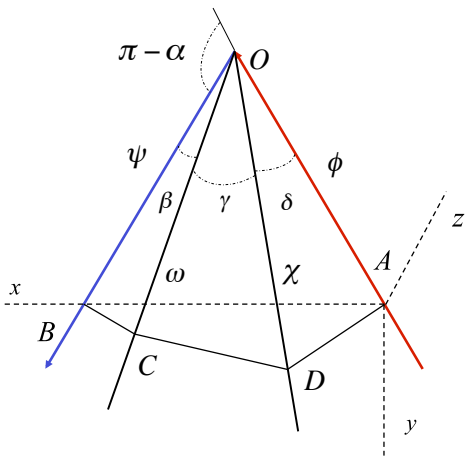

$$
\begin{aligned}
L_{1}=\cos \alpha \cos \delta, & M_{1}=\sin \alpha \sin \delta \\
L_{2}=\cos \alpha \cos \beta, & M_{2}=\sin \alpha \sin \beta \\
L_{3}=\cos \beta \cos \gamma, & M_{3}=\sin \beta \sin \gamma \\
L_{4}=\cos \gamma \cos \delta= & M_{4}=\sin \gamma \sin \delta
\end{aligned}
$$

Figure 9 A tetrahedral angle with vertex at $O$

Then

$$
\begin{array}{r}
\left(L_{1}-L_{3}-M_{1}+M_{3}\right) u^{2} w^{2}+\left(L_{1}-L_{3}-M_{1}-M_{3}\right) u^{2}+\left(L_{1}-L_{3}+M_{1}+M_{3}\right) w^{2}+\left(L_{1}-L_{3}+M_{1}-M_{3}\right)=0 \\
\left(L_{2}-L_{4}-M_{2}+M_{4}\right) v^{2} x^{2}+\left(L_{2}-L_{4}-M_{2}-M_{4}\right) v^{2}+\left(L_{2}-L_{4}+M_{2}+M_{4}\right) x^{2}+\left(L_{2}-L_{4}+M_{2}-M_{4}\right)=0
\end{array}
$$

which we rewrite as

$$
\begin{array}{r}
F_{1} u^{2} w^{2}+G_{1} u^{2}+H_{1} w^{2}+J_{1}=0 \\
F_{2} v^{2} x^{2}+G_{2} v^{2}+H_{2} x^{2}+J_{2}=0
\end{array}
$$

with

$F_{i}=L_{i}-L_{i+2}-M_{i}+M_{i+2}, G_{i}=L_{i}-L_{i+2}-M_{i}-M_{i+2}, H_{i}=L_{i}-L_{i+2}+M_{i}+M_{i+2}, J_{i}=L_{i}-L_{i+2}+M_{i}-M_{i+2}$.

Solving for $w^{2}, x^{2}$ in terms of $u, v$, we obtain

$$
w^{2}=-\frac{G_{1} u^{2}+J_{1}}{F_{1} u^{2}+H_{1}}, x^{2}=-\frac{G_{2} v^{2}+J_{2}}{F_{2} v^{2}+H_{2}} .
$$

The tetrahedral equation relating two adjacent dihedrals in a tetrahedral is discussed in detail in Bricard's Memoir ${ }^{5}$.To compute all the dihedrals in closed form in terms of one of them, we need to supplement the above equations with expressions involving the sines of the dihedrals $\omega, \chi$ so the ambiguity in the actual values of $(\omega, \chi)$ involved in Eqs. (3) may be resolved. For that purpose, we introduce the tetrahedral equations between angles $(\phi, \chi)$ and $(\psi, \omega)$, or rather their half-tangents $(u, x)$ and $(v, w)$ :

$$
\begin{array}{ll}
(\chi, \phi): & A_{1} u^{2} x^{2}+B_{1} u^{2}+2 C_{1} u x+D_{1} x^{2}+E_{1}=0 \\
(\psi, \omega): & A_{2} v^{2} w^{2}+B_{2} v^{2}+2 C_{2} v w+D_{2} w^{2}+E_{2}=0
\end{array}
$$

$$
\begin{array}{rlrl}
A_{1}=\cos \beta-\cos (\delta+\alpha+\gamma), & & A_{2}=\cos \delta-\cos (\beta+\alpha+\gamma) \\
B_{1}=\cos \beta-\cos (\delta+\alpha-\gamma), & & B_{2}=\cos \delta-\cos (\beta+\alpha-\gamma) \\
D_{1}=\cos \beta-\cos (\delta-\alpha+\gamma), & & D_{2}=\cos \delta-\cos (\beta-\alpha+\gamma) \\
E_{1}=\cos \beta-\cos (\delta-\alpha-\gamma), & E_{2}=\cos \delta-\cos (\beta-\alpha-\gamma) \\
C_{1}=C_{2}=-2 \sin \alpha \sin \gamma
\end{array}
$$


Substituting the expressions for $w^{2}$ and $x^{2}$ in Eq. (3) into Eqs. $(4,5)$, we get expressions for $x, w$ in terms of $u, v$ :

$$
\begin{aligned}
& w=-\frac{1}{2 C_{2} v}\left\{\left(B_{2} v^{2}+E_{2}\right)-\left(A_{2} v^{2}+D_{2}\right) \frac{G_{1} u^{2}+J_{1}}{F_{1} u^{2}+H_{1}}\right\} \\
& x=-\frac{1}{2 C_{1} u}\left\{\left(B_{1} u^{2}+E_{1}\right)-\left(A_{1} u^{2}+D_{1}\right) \frac{G_{2} v^{2}+J_{2}}{F_{2} v^{2}+H_{2}}\right\}
\end{aligned}
$$

We can solve Eq. (2) for $v$ in terms of $u$ if it is so desired. In the inverse kinematic problem this is not needed, as these dihedrals are determined simultaneously by solving a system of coupled equations, while the following direct expression is the basis of our analysis of five-membered rings as discussed in Section 6:

$$
v=\frac{-C u \pm \sqrt{C^{2} u^{2}-\left(A u^{2}+D\right)\left(B u^{2}+E\right)}}{A u^{2}+D}
$$

For each value of $\phi$, there correspond two possible values of $\psi$ and also of $\omega, \chi$. The latter are determined uniquely once a sign for $v$ has been chosen.

\subsection{R6B6}

The general formulation of the IK problem, allowing arbitrary pivot arrangements, was first solved by a fully algebraic method of optimal degree 16 by Lee and Liang ${ }^{6}$. Our generalization for a molecular chain of arbitrary length proceeds by first identifying the 6 rotor axes to be used for closure. The linkage is then transformed to a system of twelve orthogonal axes by affixing orthogonal connectors, the bars, between successive rotors, replacing the intervening structure. In our application, the torsions on intervening driver axes are already sampled and set to fixed values, thus rigidifying the structure between successive rotors. This orthogonal system involves axes of given lengths between successive junctions. The torsions about bars relating successive rotors are fixed by the intervening geometry. The torsions about the original rotors are computed to create a closed ring structure. The ensuing set of variables are the Denavit-Hartenberg $(\mathrm{DH})$ coordinates $^{7}$ (Figure 10). Once the transformation to $\mathrm{DH}$ coordinates is performed, the problem of determining the 6 torsions to close the ring is now cast in terms of the 6 torsions connecting the orthogonal systems through reorienting the frames $Q_{i}^{+}$and $Q_{i}^{-}$about the axes $z_{i}, i=1, \cdots, 6$ of the rotors. The common approach we follow here, due to Lee and Liang ${ }^{6}$, is to traverse the ring in two ways from a starting frame $R$ (the reference frame) and express the orientations and moments of the axes of the furthest frame from the $R$ frame (the arm, or A frame) in terms of the torsions of the two sets of intervening axes (Figure 10) along each side. Equating expressions leads to a system of 20 equations for the various components whose overdeterminacy may be exploited to efficiently eliminate the variables involved in one of the circuits in terms of the variables in the other.

The IK problem of a general system containing six oblique rotor axes involves a multitude of singularities. Some of these are intrinsic ${ }^{5}$, a consequence of sometimes subtle symmetries that render the problem insoluble by the standard methods. Others arise from the numerical method, and depend on the details of carrying out the algebraic elimination of variables. In the Lee-Liang approach ${ }^{6}$, elimination is performed in a specific order. The resulting system is then put in resultant form and the remaining variables are eliminated using an efficient method finally giving a polynomial of degree 16 in one of the torsions (actually, its half-tangent). However, the Lee-Liang algorithm may fail for special cases, even though the underlying kinematic problem has isolated, well defined solutions. The reason is rather subtle and it is related to geometrical properties of manifolds of families of lines ${ }^{8}$. Among existing implementations of the original or of the closely related method due to Raghavan and Roth ${ }^{9}$, the one due to Manocha and Canny ${ }^{10}$ uses SVD to avoid a singularity, but it involves a computationally inefficient resultant of size $24 \times 24$. Wu and Deem ${ }^{11}$ proposed modifying the algorithm ${ }^{6}$ by combinatorially trying different orders of elimination among the overdetermined system of 20 equations involving the sines/cosines of pivot torsions. Indeed, as it has also been argued by Husty ${ }^{8}$ (and as our own calculations verify), it is always possible to complete the elimination, and the algebraic failure thus appears to be caused by only considering elimination in one specific order. However, there is no a priori guarantee as to how many different trials may be needed until a nonsingular combination is found by simple trial and error.

In our own approach to elimination we avoid the combinatorial search by formulating a solution process for arriving at an optimal reduced system by using the left null vectors of the SVD of the full overdetermined system to identify the best possible set of equations for which elimination is guaranteed. A key advantage of our approach over that of Manocha and Canny ${ }^{10}$ is the derivation of an optimally sized $16 \times 16$ generalized eigenproblem with no extraneous roots, the minimum size necessary to characterize the complete set of solutions, as contrasted to the 24-degree system found in ${ }^{10}$. An example of the decomposition of the 19 -member main ring of POL into orthogonal bonds and the computation of 


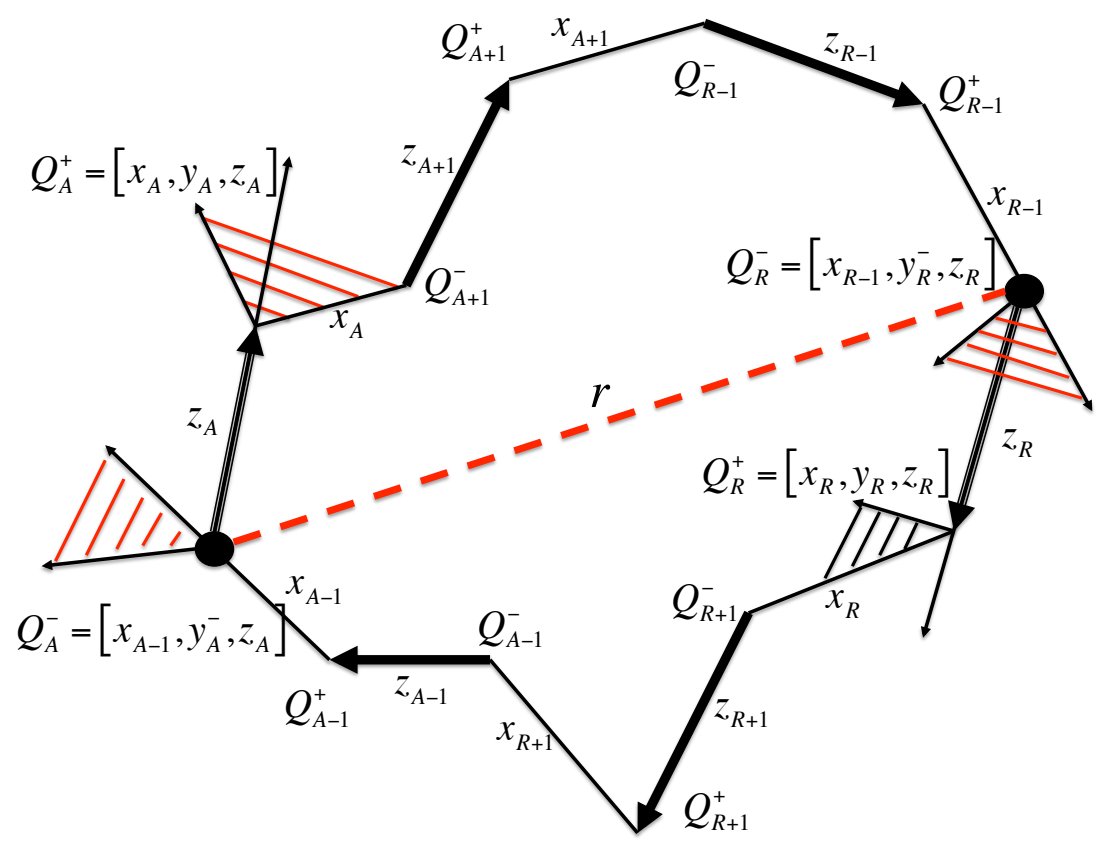

Figure 10 Coordinate systems of complete linkage showing the relationship between reference (R) and arm (A) frames.

alternative conformations by R6B6 is shown in the SI Section 2 where all non-pivot torsions are kept fixed. The technical details of the algorithm will be published elsewhere.

\section{The R6B6 algorithm: a numerical example}

We demonstrate the operation of the algorithm on a generic case, using as an example the 19-atom backbone atomic coordinates from the macrocycle polyoxypeptin A (POL). The Cartesian coordinates of the 19 atoms making up the main ring are given in Table 6 and the internal coordinates in Table 7, columns 2 and 8-9.

Figure 11 shows the decomposition into the 6R-6 Bar orthogonal linkage of the Denavit-Hartenberg representation ${ }^{7}$ and Figure 12 shows the sextet of alternative configurations found, given the particular set of rotor axes $(3,6,9,12$, $16,18)$, while keeping all other torsions fixed. Finally Tables 8-12 give the Cartesian coordinates of the five alternative structures and Table 7, columns 3-7 give the corresponding torsions. Using any of these alternative structures as input, reproduces all of the other structures as well as the original, as should be expected. The accuracy of the reconstruction is limited by the output format which includes only 3 decimal points, to be consistent with typical accuracy of PDB structures utilized by molecular structure libraries. 


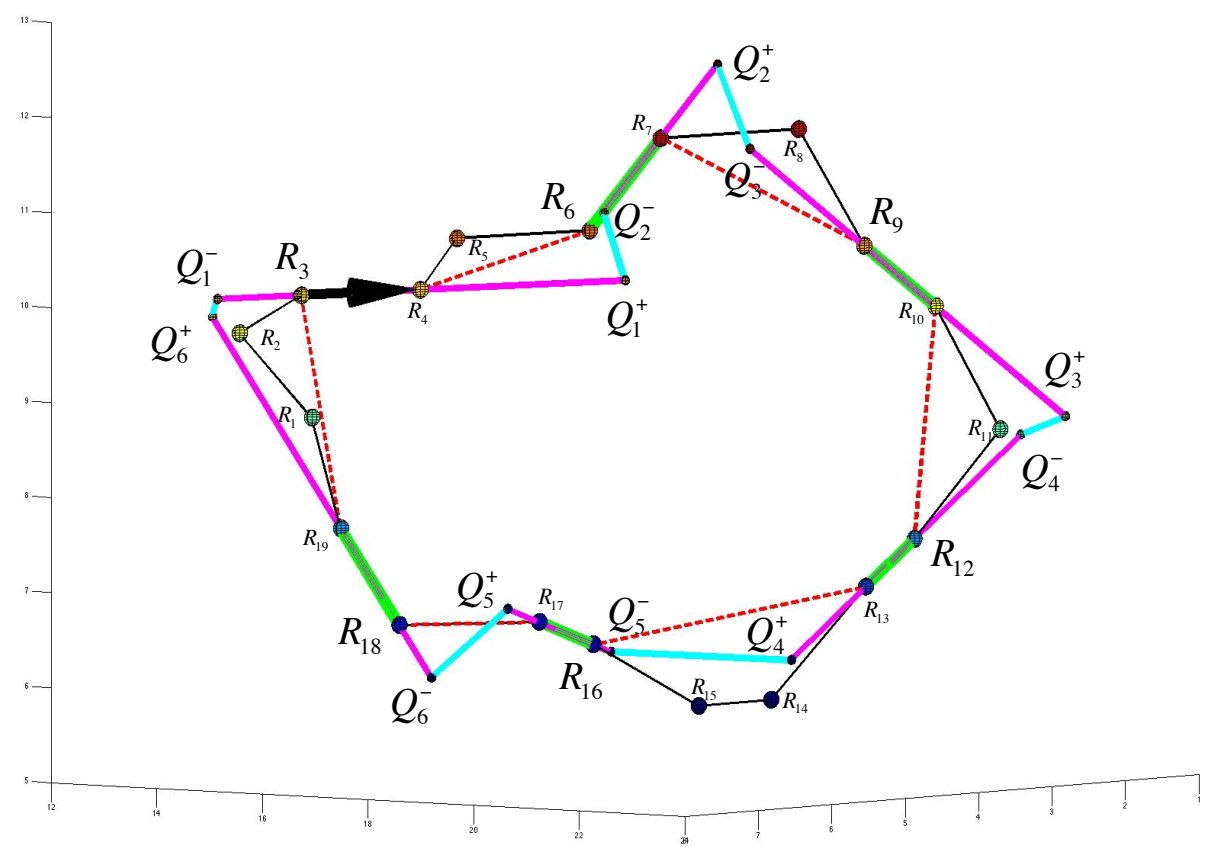

Figure 11 The orthogonal linkage for the 19-member ring of POL: rotor axes (in green) along bonds 3, 6, 9, 12, 16 and 18. Extended rotors: magenta and orthogonal linkages (bars): cyan. Broken red lines indicate virtual bonds, replacing rigid structure between rotors. Nodes in the original ring shown as large spheres. Rotor 1 is identified by a bold black arrow.

Table 6 The Cartesian coordinates for the original conformation of the 19-membered ring POL.

\begin{tabular}{c|cccc} 
Atom \# & $\mathrm{x}$ & $\mathrm{y}$ & $\mathrm{z}$ & Atom Type \\
\hline 1 & 0.000 & 0.000 & 0.000 & $\mathrm{C}$ \\
2 & 1.551 & 0.000 & 0.000 & $\mathrm{C}$ \\
3 & 2.210 & 1.332 & 0.000 & $\mathrm{~N}$ \\
4 & 1.755 & 2.563 & -0.360 & $\mathrm{C}$ \\
5 & 2.738 & 3.777 & -0.311 & $\mathrm{C}$ \\
6 & 2.108 & 5.010 & -0.822 & $\mathrm{~N}$ \\
7 & 1.902 & 5.238 & -2.141 & $\mathrm{C}$ \\
8 & 1.325 & 6.595 & -2.631 & $\mathrm{C}$ \\
9 & 0.023 & 6.964 & -2.036 & $\mathrm{~N}$ \\
10 & -0.218 & 8.143 & -1.408 & $\mathrm{C}$ \\
11 & -1.615 & 8.440 & -0.799 & $\mathrm{C}$ \\
12 & -1.881 & 7.624 & 0.400 & $\mathrm{~N}$ \\
13 & -2.599 & 6.484 & 0.490 & $\mathrm{C}$ \\
14 & -2.708 & 5.721 & 1.842 & $\mathrm{C}$ \\
15 & -3.100 & 4.301 & 1.621 & $\mathrm{~N}$ \\
16 & -2.175 & 3.322 & 1.456 & $\mathrm{C}$ \\
17 & -2.592 & 1.962 & 0.853 & $\mathrm{C}$ \\
18 & -1.897 & 0.659 & 1.348 & $\mathrm{C}$ \\
19 & -0.494 & 0.628 & 1.088 & $\mathrm{O}$
\end{tabular}




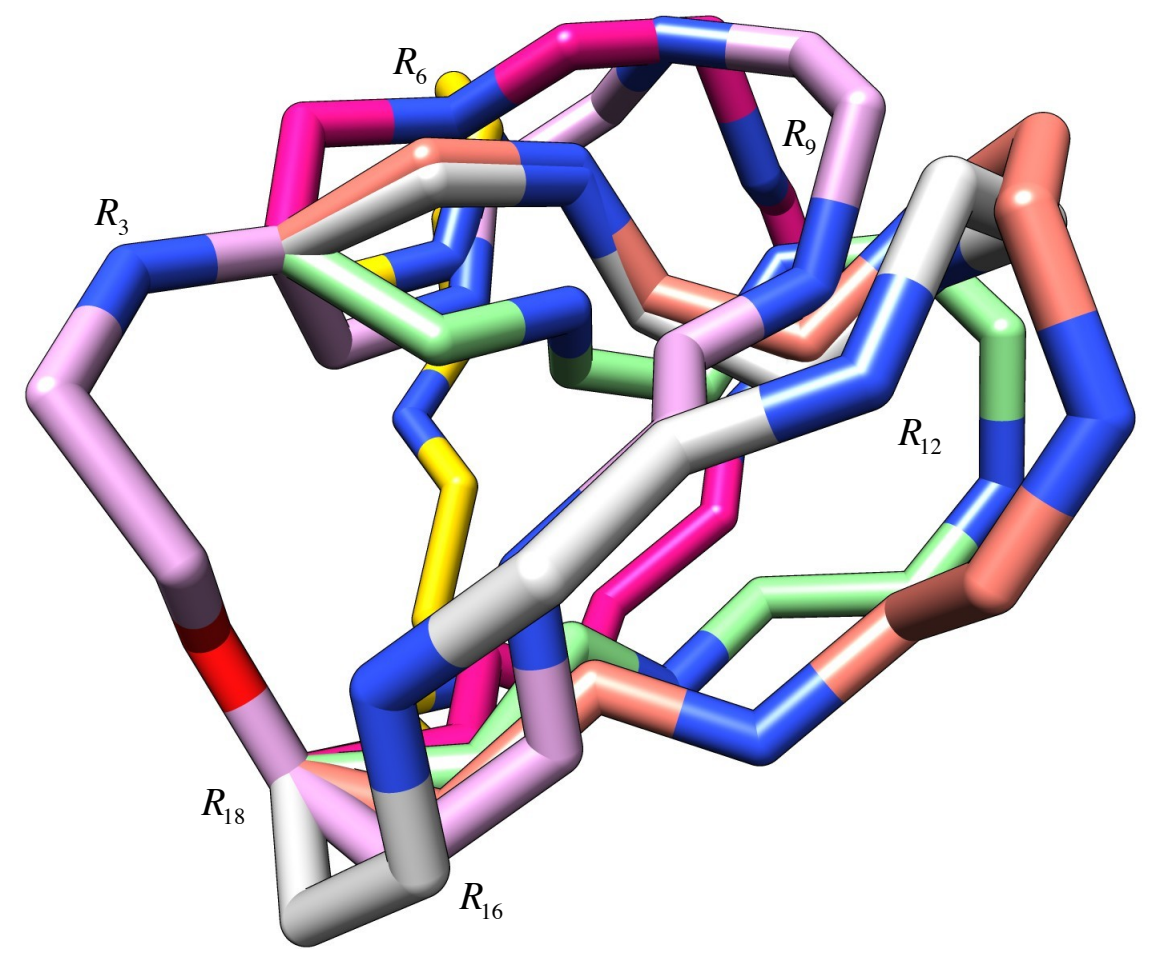

Figure 12 The 19-member main ring of POL: closed ring alternatives keeping non-pivot torsions fixed. Pivot bonds are marked by first atom ID. Original structure (red) and alternative structures 2 through 6 (respectively, yellow, violet, green, brown and grey) are superimposed with atoms 1, 2, 3, 18 and 19 held at fixed positions.

Table 7 The internal coordinates for the original and five alternative conformations of the 19-membered ring. Column 2 gives the torsions of the original structure and columns 3-7 those of alternate closed rings found by the R6B6 algorithm. All 6 rings share the same angles between successive edges (bond angles) shown in column 8 as well as edge lengths (bond lengths) shown in column 9. Non-pivot torsions are also common. Pivots are at atoms 3, 6, 9, 12, 16, 18.

\begin{tabular}{r|rrrrrrrr}
$\begin{array}{r}\text { Atom } \\
\text { Number }\end{array}$ & $\begin{array}{r}\text { Torsion } \\
\text { Solution 1 }\end{array}$ & $\begin{array}{r}\text { Torsion } \\
\text { Solution 2 }\end{array}$ & $\begin{array}{r}\text { Torsion } \\
\text { Solution 3 }\end{array}$ & $\begin{array}{r}\text { Torsion } \\
\text { Solution 4 }\end{array}$ & $\begin{array}{r}\text { Torsion } \\
\text { Solution 5 }\end{array}$ & $\begin{array}{r}\text { Torsion } \\
\text { Solution 6 }\end{array}$ & $\begin{array}{r}\text { Bond } \\
\text { Angle }\end{array}$ & $\begin{array}{r}\text { Bond } \\
\text { Length }\end{array}$ \\
\hline 1 & -60.007 & -60.007 & -60.007 & -60.007 & -60.007 & -60.007 & 111.467 & 1.551 \\
2 & -20.704 & -20.704 & -20.704 & -20.704 & -20.704 & -20.704 & 116.324 & 1.486 \\
3 & -176.866 & 112.025 & 87.382 & -5.956 & -70.168 & -63.557 & 131.491 & 1.361 \\
4 & 175.997 & 175.997 & 175.997 & 175.997 & 175.997 & 175.997 & 118.955 & 1.563 \\
5 & -75.400 & -75.400 & -75.400 & -75.400 & -75.400 & -75.400 & 111.691 & 1.476 \\
6 & -175.731 & -91.902 & 143.590 & -153.911 & -89.701 & -86.685 & 122.948 & 1.354 \\
7 & -56.654 & -56.654 & -56.654 & -56.654 & -56.654 & -56.654 & 120.733 & 1.554 \\
8 & 126.184 & 126.184 & 126.184 & 126.184 & 126.184 & 126.184 & 114.666 & 1.478 \\
9 & -179.388 & -130.200 & -126.901 & -30.598 & 0.735 & -42.002 & 124.002 & 1.358 \\
10 & 70.774 & 70.774 & 70.774 & 70.774 & 70.774 & 70.774 & 120.525 & 1.552 \\
11 & -98.760 & -98.760 & -98.760 & -98.760 & -98.760 & -98.760 & 112.087 & 1.475 \\
12 & 176.871 & -134.584 & -93.317 & 76.596 & 72.924 & -137.981 & 128.070 & 1.350 \\
13 & -159.527 & -159.527 & -159.527 & -159.527 & -159.527 & -159.527 & 120.519 & 1.557 \\
14 & 90.908 & 90.908 & 90.908 & 90.908 & 90.908 & 90.908 & 110.840 & 1.490 \\
15 & -163.246 & -163.246 & -163.246 & -163.246 & -163.246 & -163.246 & 121.771 & 1.356 \\
16 & -145.731 & 179.094 & 56.922 & -117.517 & -148.743 & 49.385 & 119.904 & 1.545 \\
17 & -62.297 & -62.297 & -62.297 & -62.297 & -62.297 & -62.297 & 119.484 & 1.558 \\
18 & -96.615 & -125.440 & -8.436 & -63.857 & -31.063 & 22.762 & 113.513 & 1.427 \\
19 & -175.146 & -175.146 & -175.146 & -175.146 & -175.146 & -175.146 & 121.110 & 1.350
\end{tabular}


Table 8 The Cartesian coordinates for the alternative conformation 2 of the 19-membered ring defined in Table 6.

\begin{tabular}{c|rrrc} 
Atom \# & $\mathrm{x}$ & $\mathrm{y}$ & $\mathrm{z}$ & Atom Type \\
\hline 1 & 0.000 & 0.000 & 0.000 & $\mathrm{C}$ \\
2 & 1.551 & 0.000 & 0.000 & $\mathrm{C}$ \\
3 & 2.210 & 1.332 & 0.000 & $\mathrm{~N}$ \\
4 & 1.755 & 2.563 & -0.360 & $\mathrm{C}$ \\
5 & 1.555 & 3.649 & 0.746 & $\mathrm{C}$ \\
6 & 1.150 & 4.946 & 0.170 & $\mathrm{~N}$ \\
7 & 2.013 & 5.768 & -0.473 & $\mathrm{C}$ \\
8 & 2.774 & 6.881 & 0.299 & $\mathrm{C}$ \\
9 & 1.906 & 7.834 & 1.022 & $\mathrm{~N}$ \\
10 & 2.028 & 8.120 & 2.344 & $\mathrm{C}$ \\
11 & 0.789 & 8.075 & 3.278 & $\mathrm{C}$ \\
12 & 0.319 & 6.696 & 3.510 & $\mathrm{~N}$ \\
13 & -0.697 & 6.049 & 2.900 & $\mathrm{C}$ \\
14 & -1.721 & 5.223 & 3.732 & $\mathrm{C}$ \\
15 & -2.422 & 4.228 & 2.873 & $\mathrm{~N}$ \\
16 & -1.950 & 2.967 & 2.710 & $\mathrm{C}$ \\
17 & -2.462 & 2.098 & 1.539 & $\mathrm{C}$ \\
18 & -1.897 & 0.659 & 1.348 & $\mathrm{C}$ \\
19 & -0.494 & 0.628 & 1.088 & $\mathrm{O}$
\end{tabular}

Table 9 The Cartesian coordinates for the alternative conformation 3 of the 19-membered ring defined in Table 6.

\begin{tabular}{c|cccc} 
Atom \# & $\mathrm{x}$ & $\mathrm{y}$ & $\mathrm{z}$ & Atom Type \\
\hline 1 & 0.000 & 0.000 & 0.000 & $\mathrm{C}$ \\
2 & 1.551 & 0.000 & 0.000 & $\mathrm{C}$ \\
3 & 2.210 & 1.332 & 0.000 & $\mathrm{~N}$ \\
4 & 1.755 & 2.563 & -0.360 & $\mathrm{C}$ \\
5 & 1.013 & 3.436 & 0.703 & $\mathrm{C}$ \\
6 & 0.675 & 4.771 & 0.172 & $\mathrm{~N}$ \\
7 & 1.592 & 5.754 & 0.013 & $\mathrm{C}$ \\
8 & 1.510 & 6.728 & -1.196 & $\mathrm{C}$ \\
9 & 1.516 & 6.071 & -2.520 & $\mathrm{~N}$ \\
10 & 0.572 & 6.279 & -3.473 & $\mathrm{C}$ \\
11 & -0.177 & 5.080 & -4.115 & $\mathrm{C}$ \\
12 & -1.117 & 4.444 & -3.171 & $\mathrm{~N}$ \\
13 & -0.928 & 3.331 & -2.431 & $\mathrm{C}$ \\
14 & -1.385 & 1.940 & -2.963 & $\mathrm{C}$ \\
15 & -1.540 & 0.969 & -1.844 & $\mathrm{~N}$ \\
16 & -2.722 & 0.806 & -1.198 & $\mathrm{C}$ \\
17 & -2.759 & 0.100 & 0.176 & $\mathrm{C}$ \\
18 & -1.897 & 0.659 & 1.348 & $\mathrm{C}$ \\
19 & -0.494 & 0.628 & 1.088 & $\mathrm{O}$
\end{tabular}


Table 10 The Cartesian coordinates for the alternative conformation 4 of the 19-membered ring defined in Table 6.

\begin{tabular}{c|cccc} 
Atom \# & $\mathrm{x}$ & $\mathrm{y}$ & $\mathrm{z}$ & Atom Type \\
\hline 1 & 0.000 & 0.000 & 0.000 & $\mathrm{C}$ \\
2 & 1.551 & 0.000 & 0.000 & $\mathrm{C}$ \\
3 & 2.210 & 1.332 & 0.000 & $\mathrm{~N}$ \\
4 & 1.755 & 2.563 & -0.360 & $\mathrm{C}$ \\
5 & 0.340 & 2.684 & -1.012 & $\mathrm{C}$ \\
6 & -0.030 & 4.093 & -1.251 & $\mathrm{~N}$ \\
7 & -0.421 & 4.929 & -0.260 & $\mathrm{C}$ \\
8 & -1.346 & 6.142 & -0.563 & $\mathrm{C}$ \\
9 & -0.826 & 7.077 & -1.582 & $\mathrm{~N}$ \\
10 & -1.516 & 7.456 & -2.688 & $\mathrm{C}$ \\
11 & -2.538 & 6.492 & -3.351 & $\mathrm{C}$ \\
12 & -3.748 & 6.315 & -2.527 & $\mathrm{~N}$ \\
13 & -4.040 & 5.302 & -1.684 & $\mathrm{C}$ \\
14 & -3.364 & 5.219 & -0.281 & $\mathrm{C}$ \\
15 & -3.432 & 3.833 & 0.259 & $\mathrm{~N}$ \\
16 & -2.447 & 2.930 & 0.035 & $\mathrm{C}$ \\
17 & -2.713 & 1.425 & 0.264 & $\mathrm{C}$ \\
18 & -1.897 & 0.659 & 1.348 & $\mathrm{C}$ \\
19 & -0.494 & 0.628 & 1.088 & $\mathrm{O}$
\end{tabular}

Table 11 The Cartesian coordinates for the alternative conformation 5 of the 19-membered ring defined in Table 6.

\begin{tabular}{c|cccc} 
Atom \# & $\mathrm{x}$ & $\mathrm{y}$ & $\mathrm{z}$ & Atom Type \\
\hline 1 & 0.000 & 0.000 & 0.000 & $\mathrm{C}$ \\
2 & 1.551 & 0.000 & 0.000 & $\mathrm{C}$ \\
3 & 2.210 & 1.332 & 0.000 & $\mathrm{~N}$ \\
4 & 1.755 & 2.563 & -0.360 & $\mathrm{C}$ \\
5 & 1.498 & 2.861 & -1.873 & $\mathrm{C}$ \\
6 & 0.919 & 4.204 & -2.074 & $\mathrm{~N}$ \\
7 & -0.375 & 4.495 & -1.802 & $\mathrm{C}$ \\
8 & -1.472 & 4.327 & -2.890 & $\mathrm{C}$ \\
9 & -1.228 & 5.086 & -4.135 & $\mathrm{~N}$ \\
10 & -1.219 & 4.531 & -5.374 & $\mathrm{C}$ \\
11 & -1.491 & 3.014 & -5.559 & $\mathrm{C}$ \\
12 & -2.901 & 2.668 & -5.297 & $\mathrm{~N}$ \\
13 & -3.436 & 2.152 & -4.170 & $\mathrm{C}$ \\
14 & -3.604 & 3.034 & -2.898 & $\mathrm{C}$ \\
15 & -3.735 & 2.187 & -1.680 & $\mathrm{~N}$ \\
16 & -2.654 & 1.816 & -0.948 & $\mathrm{C}$ \\
17 & -2.769 & 0.648 & 0.057 & $\mathrm{C}$ \\
18 & -1.897 & 0.659 & 1.348 & $\mathrm{C}$ \\
19 & -0.494 & 0.628 & 1.088 & $\mathrm{O}$
\end{tabular}


Table 12 The Cartesian coordinates for the alternative conformation 6 of the 19-membered ring defined in Table 6 .

\begin{tabular}{c|cccc} 
Atom \# & $\mathrm{x}$ & $\mathrm{y}$ & $\mathrm{z}$ & Atom Type \\
\hline 1 & 0.000 & 0.000 & 0.000 & $\mathrm{C}$ \\
2 & 1.551 & 0.000 & 0.000 & $\mathrm{C}$ \\
3 & 2.210 & 1.332 & 0.000 & $\mathrm{~N}$ \\
4 & 1.755 & 2.563 & -0.360 & $\mathrm{C}$ \\
5 & 1.350 & 2.813 & -1.849 & $\mathrm{C}$ \\
6 & 0.792 & 4.165 & -2.043 & $\mathrm{~N}$ \\
7 & -0.459 & 4.509 & -1.650 & $\mathrm{C}$ \\
8 & -1.677 & 4.272 & -2.585 & $\mathrm{C}$ \\
9 & -1.572 & 4.915 & -3.912 & $\mathrm{~N}$ \\
10 & -1.714 & 4.253 & -5.089 & $\mathrm{C}$ \\
11 & -1.075 & 2.854 & -5.290 & $\mathrm{C}$ \\
12 & -1.760 & 1.813 & -4.500 & $\mathrm{~N}$ \\
13 & -1.395 & 1.297 & -3.307 & $\mathrm{C}$ \\
14 & -1.504 & -0.233 & -3.040 & $\mathrm{C}$ \\
15 & -1.531 & -0.516 & -1.578 & $\mathrm{~N}$ \\
16 & -2.696 & -0.588 & -0.886 & $\mathrm{C}$ \\
17 & -2.682 & -0.495 & 0.656 & $\mathrm{C}$ \\
18 & -1.897 & 0.659 & 1.348 & $\mathrm{C}$ \\
19 & -0.494 & 0.628 & 1.088 & $\mathrm{O}$
\end{tabular}




\section{References}

[1] Coutsias, E. A.; Seok, C.; Dill, K. A. J. Comput. Chem. 2004, 25, 1849-1857.

[2] Coutsias, E. A.; Seok, C.; Jacobson, M. P.; Dill, K. A. A kinematic view of loop closure. J. Comput. Chem. 2004, 25, 510-528.

[3] Coutsias, E. A.; Seok, C.; Wester, M. J.; Dill, K. A. Resultants and loop closure. Int. J. Quantum Chem. 2006, 106, $176-189$.

[4] Mandell, D. J.; Coutsias, E. A.; Kortemme, T. Sub-angstrom accuracy in protein loop reconstruction by roboticsinspired conformational sampling. Nat. Methods 2009, 6, 551-552.

[5] Bricard, R. Mémoire sur la théorie de l'octaèdre articulé. J. Math. Pure Appl. 1897, 3, 113-150.

[6] Lee, H.-Y.; Liang, C.-G. Displacement analysis of the general spatial 7-link 7R mechanism. Mech. Mach. Theory 1988, 23, 219-226.

[7] Denavit, J.; Hartenberg, R. J. Appl. Mech. 1955, 77, 215-221.

[8] Husty, M. L.; Pfurner, M.; Schröcker, H.-P. A new and efficient algorithm for the inverse kinematics of the general serial 6R manipulator. Mech. Mach. Theory. 2007, 42, 66-81.

[9] Raghavan, M.; Roth, B. Kinematic analysis of the 6R manipulator of general geometry. Proceedings of the 5th International Symposium on Robotics Research (ISSR) 1990, Tokyo, 263-269.

[10] Manocha, D.; Canny, J. F. Efficient Inverse Kinematics for General 6R Manipulators. IEEE T. Robotic. Autom. 1994, $10,648-657$.

[11] Wu, M. G.; Deem, M. W. Analytical rebridging Monte Carlo: Application to cis/trans isomerization in prolinecontaining, cyclic peptides. J. Chem. Phys. 1999, 111, 6625-6632.

[12] Ho, B. K.; Coutsias, E. A.; Seok, C.; Dill, K. A. The flexibility in the proline ring couples to the protein backbone. Protein Sci. 2005, 14, 1011-1018. 